\title{
Factorization of Cyclotomic Polynomials with Quadratic Radicals in the Coefficients
}

\author{
Alfred Wünsche \\ Formerly: Institut für Physik, Humboldt-Universität, Berlin, Germany \\ Email: alfred.wuensche@physik.hu-berlin.de
}

How to cite this paper: Wünsche, A. (2017) Factorization of Cyclotomic Polynomials with Quadratic Radicals in the Coefficients. Advances in Pure Mathematics, 7, 472-506.

https://doi.org/10.4236/apm.2017.79032

Received: August 2, 2017

Accepted: September 24, 2017

Published: September 27, 2017

Copyright $\odot 2017$ by author and Scientific Research Publishing Inc. This work is licensed under the Creative Commons Attribution International License (CC BY 4.0).

http://creativecommons.org/licenses/by/4.0/

\begin{abstract}
In this article we continue the consideration of geometrical constructions of regular $n$-gons for odd $n$ by rhombic bicompasses and ruler used in [1] for the construction of the regular heptagon $(n=7)$. We discuss the possible factorization of the cyclotomic polynomial in polynomial factors which contain not higher than quadratic radicals in the coefficients whereas usually the factorization of the cyclotomic polynomials is considered in products of irreducible factors with integer coefficients. In considering the regular heptagon we find a modified variant of its construction by rhombic bicompasses and ruler. In detail, supported by figures, we investigate the case of the regular tridecagon ( $n=13$ ) which in addition to $n=7$ is the only candidate with low $n$ (the next to this is $n=769$ ) for which such a construction by rhombic bicompasses and ruler seems to be possible. Besides the coordinate origin we find here two points to fix for the possible application of two bicompasses (or even four with the addition of the complex conjugate points to be fixed). With only one bicompass one has in addition the problem of the trisection of an angle which can be solved by a neusis construction that, however, is not in the spirit of constructions by compass and ruler and is difficult to realize during the action of bicompasses. As discussed it seems that to finish the construction by bicompasses the correlated action of two rhombic bicompasses must be applied in this case which avoids the disadvantages of the neusis construction. Single rhombic bicompasses allow to draw at once two circles around two fixed points in such correlated way that the position of one of the rotating points on one circle determines the positions of all the other points on the second circle in unique way. The known case $n=17$ embedded in our method is discussed in detail.
\end{abstract}

\section{Keywords}

Geometrical Constructions by Compass and Ruler, Bicompasses, Cyclotomic Polynomials, Chebyshev Polynomials, 7-Gon, 13-Gon, 17-Gon, Fermat Numbers 


\section{Introduction}

From ancient time on it was a problem of serious and of recreational mathematics which of the regular $n$-gons may be constructed by compass and ruler (straightedge without marks) and all "simple" constructions were known without a proof of the completeness of their possibilities up to the appearance of Gauss (in German: Gauß) on the scene at the very beginning of the 19-th century. Gauss showed that the basic numbers $n$ for such constructions are the prime Fermat numbers $F_{l}=2^{2^{l}}+1,(l=0,1,2, \cdots)$ with the long known cases corresponding to $n=F_{0}=3$ and $n=F_{1}=5$ and with the first unknown and surprising case at this time $n=F_{2}=2^{2^{2}}+1=17$ (e.g., [2]-[13] and the more popular articles of Gardner [14] [15]). This results from the solution of the cyclotomic equations for these cases. A little later the general theory was developed for the solvability of polynomial equations with integer or rational coefficients in radicals (now called Galois theory) to which the cyclotomic equation is a special case. Some prehistory to this connected with names such as Lagrange, Ruffini and Abel is told by Stewart [5] (chap. 8). The construction by compass and ruler requires not higher than quadratic radicals. In [1] it was shown that the regular heptagon $(n=7)$ can be constructed by rhombic bicompasses and ruler. The rhombic bicompasses are two correlated compasses with, at least, 3 connected arms of equal length which can be fixed in two different points and which allow then the motion of the arms in two correlated circles around the fixed points with one degree of freedom. The addition of such bicompasses as device for geometric constructions is, in our persuasion, certainly in the spirit of the ancient geometers and extends our possibilities for constructions. Exact constructions with rhombic bicompasses are possible if the fixed points are determined by not higher than quadratic radicals (nested square roots) and, therefore, are constructible by compass and ruler. To apply rhombic bicompasses and ruler for the construction of regular $n$ gons it is necessary that the cyclotomic polynomials $\frac{z^{n}-1}{z-1}$ can be factorized into products of polynomial equations of 3-rd degree with not higher than quadratic radicals in their coefficients. In Sections 3, 9 and 11 we suggest arguments that this restricts the possible applications for odd $n$ to prime numbers $n$ equal to $n=G_{l}=3 \cdot 2^{2^{l}}+1$ in analogy to the Fermat numbers $F_{l}$ that means to $n=7$ for $l=0$ and next to $n=13$ for $l=1$ and discuss these cases in detail.

In present article we investigate the factorizations of the cyclotomic polynomials for low odd $n$ (up to $n=19$ ) in polynomial factors of 3 -cycles (polynomial equations of 3-rd degree with 3 involved roots) which contain not higher than quadratic radicals in the coefficients and explain how this can be obtained in explicit form. We also give in explicit form for low $n$ the factorization with only quadratic radicals in the coefficients but with other than 3cycles and determine some general rules for this. For odd order 
$n=2 m+1,(m=1,2, \cdots)$ in a first step the factorization of the cyclotomic polynomial in a product of two polynomials of $m$-th degree with not higher than quadratic radicals in the coefficients is generally possible. Concerning the regular heptagon $(n=7)$ we add a further modification of its construction by rhombic bicompasses and ruler and we discuss in detail the interesting case $n=13$ where some problems remain open. For the well-known case $n=17$ we find in fully explicit form the factorization of the cyclotomic polynomial in 8- 4and 2-cycles with nested quadratic radicals in the coefficients. For odd and even $n$ we express the the polynomials for the determination of the Cosines of the angles of the circle-division problem by Chebyshev polynomials of first and second kind and derive more information about this in an appendix.

The cyclotomic polynomials $p_{n}(z)$ for the complex corner points of a regular $n$-gon to circumradius $r=1$ at the coordinate origin as it is well known are

$$
p_{n}(z)=z^{n}-1, \quad\left(z \equiv x+\mathrm{i} y \equiv(x, y), z^{*} \equiv x-\mathrm{i} y \equiv(x,-y)\right) .
$$

The $n$ complex solutions $z=z_{k}=\exp \left(\mathrm{i} k \frac{2 \pi}{n}\right),(k=0,1,2, \cdots$, modulo $n)$ of the cyclotomic equation

$$
0=z^{n}-1=(z-1)\left(z^{n-1}+z^{n-2}+\cdots+z+1\right), \quad\left(z_{0} \equiv z_{n}=1\right),
$$

solve the problem of the circle division into $n$ equal sectors and determine the corner points

$$
\begin{aligned}
& z_{k} \equiv \exp \left(\mathrm{i} k \frac{2 \pi}{n}\right), \quad z_{k}^{-1}=z_{k}^{*}, \\
& z_{k} z_{l}=z_{k+l}=z_{1}^{k+l}, \quad(k=0,1,2, \cdots,(\bmod n)),
\end{aligned}
$$

of the regular $n$-gon in the complex plane.

In the following we describe the procedure to obtain factorizations of the cyclotomic equation with not higher than quadratic radicals in the coefficients and give the explicit results for odd $n$ up to $n=19$. In particular, we discuss in detail the cases $n=7$ and $n=13$ which possess a relation to the application of bicompasses and ruler. In the case $n=17$ which we also discuss in some detail we demonstrate how our method acts in a case known since Gauss. The results for all corner points of the regular 17-gon are given in an explicit form (see and compare also [9] [11] [12]).

\section{The Cosine of the Angles for the Cyclotomic Polynomials in Odd Case $n=2 m+1$}

We consider in this Section the case of the Cosine of the angles for the odd case $n=2 m+1,(m=1,2, \cdots)$ of the regular $n$-gon and introduce the Cosine of angles for $z$ on the unit circle by

$$
x=\frac{z+z^{-1}}{2}=\frac{z+z^{*}}{2} \equiv \cos (\theta) .
$$


The cyclotomic polynomials $p_{2 m+1}(z)$ can be transformed to

$$
\begin{aligned}
\frac{p_{2 m+1}(z)}{(z-1) z^{m}} & =z_{0}+\sum_{k=1}^{m}\left(z^{k}+z^{-k}\right)=1+2 \sum_{k=1}^{m} \cos (k \theta)=1+2 \sum_{k=1}^{m} \mathrm{~T}_{k}(\cos (\theta)) \\
& =1+2 \sum_{k=1}^{m} \mathrm{~T}_{k}(x)=\mathrm{U}_{m}(x)+\mathrm{U}_{m-1}(x)
\end{aligned}
$$

where $\mathrm{T}_{n}(x)$ are the Chebyshev polynomials of first kind and $\mathrm{U}_{n}(x)$ the Chebyshev polynomials of second kind (e.g., [16] [17] [18]). The well-known property of the Chebyshev polynomials

$$
\mathrm{T}_{n}(\cos (\theta))=\cos (n \theta), \quad \mathrm{U}_{n}(\cos (\theta))=\frac{\sin ((n+1) \theta)}{\sin (\theta)},
$$

is used. The relation between $\mathrm{U}_{n}(x)$ and $\mathrm{T}_{n}(x)$ in the second line of (2.2) together with many other relations for the Chebyshev polynomials may be proved by complete induction using the addition theorems for the trigonometric functions.

For a few first polynomials $\mathrm{U}_{m}(x)+\mathrm{U}_{m-1}(x)$ concerning the odd regular $n$-gons $(n=2 m+1)$ one finds explicitly together with possible factorizations with integer or rational coefficients (i.e. in $\mathbb{Z}$ or $\mathbb{Q}$ ) in case of prime or composite $n$ :

One peculiarity is that the polynomials $\mathrm{U}_{m}(x)+\mathrm{U}_{m-1}(x)$ take on their simplest form with lowest integer coefficients by the substitution $x=u / 2$ (similarly to the polynomials $\mathrm{U}_{m}(x)$ themselves; but not $\mathrm{T}_{n}(x)$ ). These polynomials possess factorizations with integer coefficients for composite numbers $n=2 m+1$. The polynomials $\mathrm{U}_{n}(z)+\mathrm{U}_{n-1}(z)$ are sometimes denoted by $\mathrm{V}_{n}(z)$ (see Appendix A).

The Cosines $\cos \left(k \theta_{1}\right)=\cos \left(k \frac{2 \pi}{n}\right)$ of the angles $k \theta_{1}$ are obtained from the solutions $x=\cos (\theta)$ of the equation

Table 1. Cyclotomic polynomials for odd $n$ and variable $x=\cos (\theta) \equiv \frac{u}{2}$ and integer factorization.

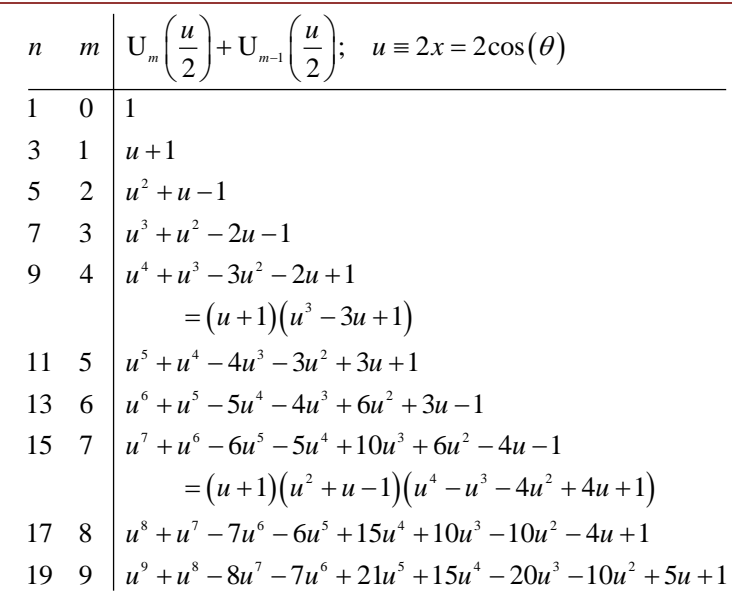




$$
\frac{p_{2 m+1}(z)}{(z-1) z^{m}}=\mathrm{U}_{m}(x)+\mathrm{U}_{m-1}(x)=0, \quad\left(x \equiv \frac{z+z^{*}}{2} \equiv \cos (\theta)\right),
$$

with exclusion of the solution $z_{0}=x_{0}=1$. From the geometrical meaning of the polynomials as providing the doubled Cosines of the angle division problem as roots for odd $n=2 m+1$ within the unit circle it is fully obvious that all roots of all polynomials in (2.4) possess only $m$ real solutions within the limits $-1 \leq x=u / 2 \leq 1$.

Without further going into details we mention that in case of even numbers $n=2 m+2$ the cyclotomic polynomials for the Cosines of the angles $k \theta$ can be represented in the form (we use $\mathrm{T}_{-k}(x)=\mathrm{T}_{k}(x)$ )

$$
\begin{aligned}
\frac{p_{2 m+2}(z)}{\left(z^{2}-1\right) z^{m}} & =\sum_{l=0}^{m} z^{m-2 l}=\sum_{l=0}^{m} \frac{z^{m-2 l}+z^{2 l-m}}{2}=\sum_{l=0}^{m} \cos ((m-2 l) \theta) \\
& =\sum_{l=0}^{m} \mathrm{~T}_{m-2 l}(\cos (\theta))=\sum_{l=0}^{m} \mathrm{~T}_{m-2 l}(x)=\mathrm{U}_{m}(x), \quad\left(x \equiv \frac{z+z^{*}}{2} \equiv \cos (\theta)\right) .
\end{aligned}
$$

The vanishing of these polynomials provides as solutions the possible Cosines of the angles to the corners of the $n$-gons with even $n=2 m+2$ with exclusion of the already eliminated Cosines $x_{0}=1, x_{m+1}=-1$.

We mention here that as (irreducible) cyclotomic polynomials $\Phi_{n}(z)$ are mostly understood the polynomials $p_{n}(z)=z^{n}-1$ divided by all products of (irreducible) polynomials $\Phi_{d}(z)$ where $d<n$ runs over all divisors of $n$ (i.e., irreducible in the sense of coefficients in $\mathbb{Q}$ or in $\mathbb{Z}$ but likely here already in $\mathbb{Z}$ ) (e.g., van der Waerden [3], Stillwell [7], Shkolnik [11] (p. 40)). They are for $n \geq 2$ palindromic polynomials (only $\Phi_{1}(z)=z-1$ is not palindromic) with real coefficients ${ }^{1}$. General explicit formulae for the polynomials $\Phi_{n}(z)$ seem to be possible for different divisibility classes and for prime $n=p$ it is $\Phi_{n}(z)=\frac{p_{n}(z)}{z-1}=\sum_{k=0}^{p-1} z^{k}$ that means they are then of degree $p-1$. Stillwell [7] (p. 70) mentions as a curious property of the polynomials $\Phi_{n}(z)$ that the first polynomial $\Phi_{n}(z)$ with coefficients of modulus 2 besides 1 (and 0 ) is $\Phi_{105}(z)$ of degree 48 whereas all polynomials $\Phi_{n}(z)$ with $n<105$ possess coefficients only of modulus 1 or equal to $0^{2}$.

In connection with constructions by rhombic bicompasses and ruler we are mainly interested in factorizations of the polynomials $\frac{p_{n}(z)}{z-1}$ with not higher than (in general, nested) quadratic radicals in the coefficients. A general explicit ${ }^{1}$ By definition, a polynomial $P_{m}(z)=\sum_{k=0}^{m} a_{k} z^{k}$ is palindromic if for all coefficients holds $a_{k}=a_{m-k}$. ${ }^{2}$ With a PC and program Mathematica one may astonishingly easily and quickly calculate the polynomials $\Phi_{n}(z)$ for "high" $n$ in explicit form by factorization of $p_{n}(z)=z^{n}-1$. It seems that such polynomials with coefficients of modulus $\neq 1$ preferably appear if the composite numbers $n$ are products of primes $3,5,7,11, \ldots$ (e.g, $n=105=3 \times 5 \times 7$ ) but not for all such products (not besides for $n<105$, e.g., for $n=231$ but for, e.g., $n=165,195,385$ ). For $n=1155=3 \times 5 \times 7 \times 11$ the polynomial $\Phi_{1155}(z)$ contains a lot of coefficients of modulus $1,2,3$ or equal to 0 and is of degree 480. 
formula for the factorized polynomials could not be obtained but a procedure will be described how such factorization in given cases leads to the result.

\section{Cycles in the Circle-Division Problem}

We explain in this Section the factorization of the cyclotomic equation for odd $n=2 m+1$ and concentrate us to the case $n=p$ where $p$ is a prime number larger than $p=2$. A solution $z_{k}=\exp \left(\mathrm{i} k \frac{2 \pi}{n}\right)$ of the cyclotomic equation $z^{n}-1=0$ is called a primitive root if there does not exist a positive integer $m<n$ for which $z_{k}^{m}=1$. It is clear that in each case $z_{1}=\exp \left(i \frac{2 \pi}{n}\right)$ is a primitive root and that for prime numbers $n=p$ all solutions $z_{k}$ with $k=1,2, \cdots, n-1,(\bmod n)$ are primitive roots (only $z_{0}=1$ is never primitive). For prime $n=p$ the cyclotomic polynomial in the form $\frac{p_{n}(z)}{z-1}$ cannot be factorized into polynomials with integer coefficients. It is said that it is irreducible in $\mathbb{Q}$ and therefore also in $\mathbb{Z}$ with coefficient of the highest power equal to 1 . Obviously, this does not mean that it is not factorizable into polynomials with radicals in the coefficient from which such are interesting for us which contain not higher than quadratic radicals since such radicals are constructible by compass and ruler. To obtain such factorizations one may apply a procedure using the little theorem of Fermat. We explain this in the following.

According to the little theorem of Fermat (e.g., [3] [8] [9]) for prime numbers $p$ and natural numbers $g=1,2, \cdots,(\bmod p)$ holds $($ symbol $\equiv$ stands here for congruences modulo $p$ )

$$
g^{p-1} \equiv 1, \quad(\bmod p) .
$$

This means that for prime numbers $n=p$ and for the primitive root $z_{1}$ we have

$$
z_{1}^{g^{n-1}}=z_{g^{n-1}}=z_{1}, \quad \Rightarrow z_{k}^{g^{n-1}}=z_{1}^{k g^{n-1}}=z_{1}^{k}=z_{k} .
$$

We choose positive integers $g=1,2, \cdots, n-1$ and form first with the solution $z_{1}$ the sequences $\left(z_{1}^{g^{0}}=z_{g^{0}}=z_{1}, z_{1}^{g^{1}}=z_{g^{1}}, z_{1}^{g^{2}}=z_{g^{2}}, \cdots, z_{1}^{g^{l}}=z_{g^{l}}\right)$ up to the case when $z_{1}^{g^{l+1}}=z_{g^{l+1}}=z_{1},(\bmod n)$. Each such sequence we call a cycle. The sequence $\left(z_{1}, Z_{g^{1}}, Z_{g^{2}}, \cdots, z_{g^{n-1}}\right)$, latest after the next step to $z_{g^{n}}$, leads back to the initial solution $z_{1}$. If $l$ is a divisor of $n-1=2 m$ then, depending on the choice of $g$, the mentioned sequence may lead back to $z_{1}$ already after $l+1$ steps. For odd prime $n=2 m+1$ the numbers $l_{1}=2, l=2 m$ and $l_{2}=m$ are divisors of $n-1=2 m$ and there are sequences with cycle lengths 2 belonging to the choice $g=2 m$ and such with cycle lengths $m$ and $2 m$. If one does not begin with the element $z_{1}$ in a cycle but with another element of this cycle then one obtains by the described procedure the same cycle with rotated order of the elements. If a root $z_{k}$ is not contained in a certain cycle then we form the 
sequences $\left(z_{k}^{g^{0}}=z_{k g^{0}}=z_{k}, z_{k}^{g^{1}}=z_{k g}, z_{k}^{g^{2}}=z_{k g^{2}}, \cdots, z_{k}^{g^{l}}=z_{k g^{l}}\right)$ and obtain for prime $n$ an equivalent cycle of the same length and so we may continue up to the case when all numbers $z_{k} \neq z_{0},(\bmod n$ are comprised.

We illustrate the factorization in cycles for our two most interesting prime cases $n=7$ and 13 :

1. Case $n=7$, basic cycles (all equalities are modulo 7 in the indices)

$$
\begin{gathered}
g=1: z_{1},\left(z_{1}=z_{1}\right), \\
g=2: z_{1}, z_{2}, z_{4},\left(z_{8}=z_{1}\right), \\
g=3: z_{1}, z_{3}, z_{9}=z_{2}, z_{6}, z_{18}=z_{4}, z_{12}=z_{5},\left(z_{15}=z_{1}\right), \\
g=4: z_{1}, z_{4}, z_{16}=z_{2},\left(z_{8}=z_{1}\right), \\
g=5: z_{1}, z_{5}, z_{25}=z_{4}, z_{20}=z_{6}, z_{30}=z_{2}, z_{10}=z_{3},\left(z_{15}=z_{1}\right), \\
g=6: z_{1}, z_{6},\left(z_{36}=z_{1}\right) .
\end{gathered}
$$

For $g=3$ and $g=5$ the sequences are 6-cycles which comprise all $n-1$ solutions $z_{k} \neq z_{0}=1$ in different order of the elements. For $g=2$ and $g=4$ we find equivalent 3 -cycles in different order of the elements $\left(z_{1}, z_{2}, z_{4}\right)$ which can be complemented by the 3-cycle $\left(z_{3}, z_{6}, z_{5}=z_{12}\right)$ to comprise all elements $z_{k} \neq z_{0}$. For $g=n-1=6$ we find the 2-cycle $\left(z_{1}, z_{6}\right)$ which can be complemented by the other possible 2-cycles $\left(z_{2}, z_{5}\right)$ and $\left(z_{3}, z_{4}\right)$ for which one does not find a factorization in polynomials of 2-nd degree with only quadratic radicals in the coefficients. In the trivial case $g=1$ one finds in every case only the 1-cycle with element $\left(z_{1}\right)$ which can be complemented by 1 -cycles $\left(z_{k}\right)$ of the other roots.

2. Case $n=13$, basic cycles (all equalities are modulo 13 in the indices)

$$
\begin{gathered}
g=1: z_{1},\left(z_{1}=z_{1}\right), \\
g=2: z_{1}, z_{2}, z_{4}, z_{8}, z_{16}=z_{3}, z_{6}, z_{12}, z_{24}=z_{11}, z_{22}=z_{9}, \\
z_{18}=z_{5}, z_{10}, z_{20}=z_{7},\left(z_{14}=z_{1}\right), \\
g=3: z_{1}, z_{3}, z_{9},\left(z_{27}=z_{1}\right), \\
g=4: z_{1}, z_{4}, z_{16}=z_{3}, z_{12}, z_{48}=z_{9}, z_{36}=z_{10},\left(z_{40}=z_{1}\right), \\
g=5: z_{1}, z_{5}, z_{25}=z_{12}, z_{60}=z_{8},\left(z_{40}=z_{1}\right), \\
g=6: z_{1}, z_{6}, z_{36}=z_{10}, z_{60}=z_{8}, z_{48}=z_{9}, z_{54}=z_{2}, z_{12}, z_{72}=z_{7}, \\
z_{42}=z_{3}, z_{18}=z_{5}, z_{30}=z_{4}, z_{24}=z_{11},\left(z_{66}=z_{1}\right), \\
g: z_{1}, z_{7}, z_{49}=z_{10}, z_{70}=z_{5}, z_{35}=z_{9}, z_{63}=z_{11}, z_{77}=z_{12}, z_{84}=z_{6}, \\
z_{42}=z_{3}, z_{21}=z_{8}, z_{56}=z_{4}, z_{28}=z_{2},\left(z_{14}=z_{1}\right), \\
g=8: z_{1}, z_{8}, z_{64}=z_{12}, z_{96}=z_{5},\left(z_{40}=z_{1}\right), \\
g=9: z_{1}, z_{9}, z_{81}=z_{3},\left(z_{27}=z_{1}\right), \\
g=10: z_{1}, z_{10}, z_{100}=z_{9}, z_{90}=z_{12}, z_{120}=z_{3}, z_{30}=z_{4},\left(z_{40}=z_{1}\right),
\end{gathered}
$$




$$
\begin{gathered}
g=11: z_{1}, z_{11}, z_{121}=z_{4}, z_{44}=z_{5}, z_{55}=z_{3}, z_{33}=z_{7}, z_{77}=z_{12}, z_{132}=z_{2}, \\
z_{22}=z_{9}, z_{99}=z_{8}, z_{88}=z_{10}, z_{110}=z_{6},\left(z_{66}=z_{1}\right), \\
g=12: z_{1}, z_{12},\left(z_{144}=z_{1}\right) .
\end{gathered}
$$

We have here cycles of lengths 1,2,3,4,6,12 which all are divisors of $n-1=2 m=12$. For example, we find the following four 3-cycles

$\left\{\left(z_{1}, z_{3}, z_{9}\right),\left(z_{4}, z_{10}, z_{12}\right)\right\},\left\{\left(z_{2}, z_{5}, z_{6}\right),\left(z_{7}, z_{8}, z_{11}\right)\right\}$ covering all primitive roots where the two pairs of 3-cycles in braces form two 6-cycles. The 3-cycles follow from the subdivision of the 12-cycles in two step by division of 2 leading first to two 6-cycles and then in last step by division of 2 to four 3-cycles. The subdivision of the cyclotomic equation of 12 -th degree in a product of six 2 cycles with quadratic equations containing 6 paired roots

$\left(z_{k}, z_{-k}=z_{k}^{*} \equiv z_{13-k},(k=1,2, \cdots, 6)\right)$ does not lead to the explicit form of the quadratic equations since the resolution of the 12-degree cyclotomic polynomial in one step by division of 3 is not possible with coefficients in form of quadratic radicals independently from the order in which the division by 3 is made, from $12 \rightarrow 4 \rightarrow 2$ or $12 \rightarrow 6 \rightarrow 2$. Therefore the three 4 -cycles

$\left(z_{1}, z_{5}, z_{8}, z_{12}\right),\left(z_{2}, z_{3}, z_{10}, z_{11}\right),\left(z_{4}, z_{6}, z_{7}, z_{9}\right)$ obtained from choice $g=5$ and $g=8$ are also not to find in form of polynomial equations with only quadratic radicals as coefficient. Each of these three 4-cycles contains only one of the roots of the four 3-cycles.

If we look to the cycles in (3.3) and (3.4) we find in case of $g \neq 1$ for the sum of the powers of $g$ within the cycle

$$
\sum_{j=0}^{l} g^{j}=\frac{g^{l+1}-1}{g-1} \equiv n, \bmod n, \quad(g \neq 1) .
$$

This is a general property which we will prove now. According to the definition of a cycle of length $l$ the power $g^{l}$ is the last in the cycle before the next power $g^{l+1}$ leads back the root $z_{1}$ to $z_{g^{l+1}}=z_{1}$ and $g^{l+1}$ is congruent to 1 modulo $n$. Since the sum on the left-hand side in (3.5) is a positive integer and $g-1 \neq 0$ is also a positive integer the right-hand side in (3.5) is a positive integer and due to the given congruence a multiple of $n$. From this follows for the product of roots within a cycle with the primitive root $z_{1}$

$$
\prod_{j=0}^{l} z_{g^{j}}=z_{1} z_{g^{\prime}} z_{g^{2}} \cdots z_{g^{l}}=z_{1}^{\sum_{j=0}^{l} g^{j}}=z_{1}^{\frac{g^{l+1}-1}{g-1}}=z_{\frac{g^{l+1}-1}{g-1}}=z_{0}=1 .
$$

The same is the case with each cycle of the length $l$ containing an arbitrary primitive root $z_{k}$. A consequence is that we know at once the constant term in the factorized cyclotomic polynomials that is of importance when we begin from behind (low-order $k$-terms proportional to $z^{k}$ ) to find the factor polynomials in factorizations.

The question about the cyclotomic polynomials $\frac{p_{2 m+1}(z)}{z-1}$ of degree $2 m$ 
Table 2. Fermat numbers $F_{l}$ and related numbers $G_{l}$ and factorization into prime numbers.

\begin{tabular}{ccccccccc}
\hline$l$ & 0 & 1 & 2 & 3 & 4 & 5 \\
& & & & & & & & \\
$F_{l} \equiv 2^{2^{l}}+1$ & 3 & 5 & 17 & 257 & 65537 & 4294967297 & $(3.7)$ \\
prime factors & & & & & & $641 \cdot 6700417$ \\
$G_{l} \equiv 3 \cdot 2^{2^{\prime}}+1$ & 7 & 13 & 49 & 769 & 196609 & 12884901889 \\
prime factors & & & $7^{2}$ & & $7 \cdot 28087$ & $19^{2} \cdot 35692249$ \\
\hline
\end{tabular}

which can be split into products of polynomials of 3-rd degree (3-cycles) leads basically, analogously to Fermat numbers $F_{l}=2^{2^{1}}+1$, to numbers

$G_{l}=3 \cdot 2^{2^{l}}+1$ which have to be prime numbers. Table 2 shows the few initial possibilities up to $l=5$.

For the next three cases $l=6,7,8$ the numbers $F_{l}$ and $G_{l}$ are composite numbers as the computer shows but these numbers grow very fast and my PC (with program "Mathematica 10") did not provide a result for the next case $l=9$ of both numbers in acceptable time. However, it is now known that all numbers $F_{l}$ from $l=9$ up to $l=19$ are composite without knowing all prime factors in all these cases (see [9], end of chap. 5). Since the number $G_{2}=49$ is composite it is not a possible candidate for construction of the regular 49-gon by rhombic bicompasses and ruler.

We mention here that if one admits angle trisection by a neusis construction attributed to Archimedes [2] [9] as an additional element of constructions which in our persuasion is not in the spirit of ancient constructions by compass and ruler then one comes to possible numbers for the solubility of the circle division problem of the form $P_{k, l}=2^{k} 3^{l}+1,(k, l=0,1,2, \cdots)$ if they are prime numbers and which are called Pierpont numbers (from 1895, see [19] [20]). These numbers are more general ones than the Fermat numbers $F_{l}$ and also than the numbers $G_{l}$ in Table 2 (see also end of Section 11).

\section{Factorization of Cyclotomic Polynomial for $\boldsymbol{n}=\mathbf{3}$ with Real Coefficients}

In case of $n=3$ the cyclotomic polynomial $\frac{p_{3}(z)}{z-1}$ can be represented

$$
\frac{z^{3}-1}{z-1}=z^{2}+z+1=\left(z+\frac{1-i \sqrt{3}}{2}\right)\left(z+\frac{1+i \sqrt{3}}{2}\right)=\left(z-z_{1}\right)\left(z-z_{2}\right) .
$$

It is written down here for the analogy to higher less trivial cases.

\section{Factorization of Cyclotomic Polynomial for $\boldsymbol{n}=\mathbf{5}$ with Quadratic Radicals}

In case of $n=5$ the cyclotomic polynomial $\frac{p_{5}(z)}{z-1}$ possesses the factorization with two 2-cycles which has the form 


$$
\begin{aligned}
\frac{z^{5}-1}{z-1} & =z^{4}+z^{3}+z^{2}+z+1 \\
& =\left(z^{2}+\frac{1-\sqrt{5}}{2} z+1\right)\left(z^{2}+\frac{1+\sqrt{5}}{2} z+1\right) \\
& =\left(\left(z-z_{1}\right)\left(z-z_{4}\right)\right)\left(\left(z-z_{2}\right)\left(z-z_{3}\right)\right) .
\end{aligned}
$$

The complex conjugate roots $\left(z_{1}, z_{4}\right)$ and $\left(z_{2}, z_{3}\right)$ modulo 5 are here paired in one of the two 2-cycles and therefore the coefficients in form of quadratic radicals possess real values. The case of the regular pentagon is also commonly known and we do not consider it in detail.

\section{Factorization of Cyclotomic Polynomial for $\boldsymbol{n}=7$ with Quadratic Radicals}

In case of $n=7$ the cyclotomic polynomial $\frac{p_{7}(z)}{z-1}$ possesses the following factorization with two 3-cycles with only quadratic radicals in the coefficients

$$
\begin{aligned}
\frac{z^{7}-1}{z-1} & =z^{6}+z^{5}+z^{4}+z^{3}+z^{2}+z+1 \\
& =\left(z^{3}+\frac{1-\mathrm{i} \sqrt{7}}{2} z^{2}-\frac{1+\mathrm{i} \sqrt{7}}{2} z-1\right)\left(z^{3}+\frac{1+\mathrm{i} \sqrt{7}}{2} z^{2}-\frac{1-\mathrm{i} \sqrt{7}}{2} z-1\right) \\
& =\left(\left(z-z_{1}\right)\left(z-z_{2}\right)\left(z-z_{4}\right)\right)\left(\left(z-z_{3}\right)\left(z-z_{5}\right)\left(z-z_{6}\right)\right) .
\end{aligned}
$$

The factorization in this case with only quadratic radicals in the coefficients and concerning the corners of the regular heptagon was discussed in [1]. It is easy to determine this factorization with quadratic radicals in the coefficients from $z_{1}+z_{2}+z_{4}=-\frac{1}{2}+\mathrm{i} y$ and $z_{6}+z_{5}+z_{3}=z_{1}^{*}+z_{2}^{*}+z_{4}^{*}=-\frac{1}{2}-\mathrm{i} y$ with imaginary party to find from the polynomial using $\sum_{k=1}^{6} z_{k}=-1$ and $z_{1} z_{2} z_{4}=z_{1}^{1+2+4}=1$. The "primitive" roots $z_{k},(k=1, \cdots, 6)$ of the polynomials (6.1) form two 3-cycles $\left(z_{1}, z_{2}, z_{4}\right)$ and $\left(z_{6}, z_{12}=z_{5}, z_{24}=z_{10}=z_{3}\right)$ modulo 7 in the indices. The quadratic radical for the sum $z_{1}+z_{2}+z_{4}$ together with the circle division in 7 equal parts is shown in Figure 1.

In Figure 2 we illustrate possibilities for the construction of the regular heptagon by bicompasses and ruler and draw additionally a circle with radius $r_{2}=\sqrt{2}$ around the coordinate origin $(0,0)$ which is equal to the distance from the coordinate origin to the fixed point $\left(-\frac{1}{2}, \frac{\sqrt{7}}{2}\right)$ of the bicompasses. This circle possesses intersection points with the circle of radius $r_{1}=1$ around the mentioned second fixpoint $\frac{-1+\mathrm{i} \sqrt{7}}{2}$ at $\mathrm{z}=\frac{1+\mathrm{i} \sqrt{7}}{2}=1+z_{1}+z_{2}+z_{4}$ and at $z=\frac{-5+i \sqrt{7}}{4}$ in the complex plane as one easily calculates. In this way we see that the intersection point $\frac{1+\mathrm{i} \sqrt{7}}{2}$ lies on the second arm of the absolute mini- 


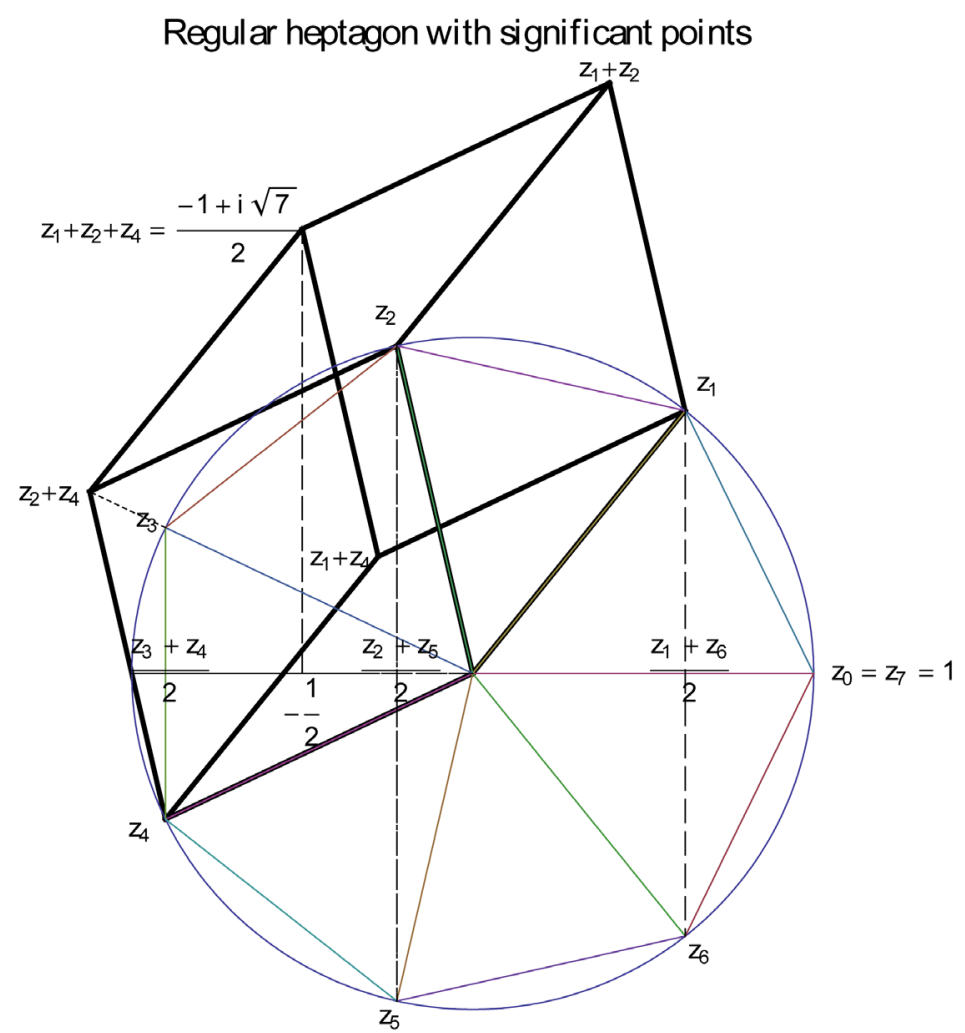

Figure 1. Regular heptagon with axes projection of corners in complex $z$-plane. Besides the coordinate origin $z=0$ the second fixed point for the rhombic bicompasses is $z=\frac{-1+\mathrm{i} \sqrt{7}}{2}$ (figure from [1] made by "Mathematica 6 ").

mum 3-arm bicompasses (see [1]) in the right position for the construction of the regular heptagon that means on the line between $z_{1}$ and $z_{1}+z_{2}$. Clearly, one has not only to believe to the optical impression but have to prove it.

The line between $z_{1}$ and $z_{1}+z_{2}$ can be parameterized by

$$
z=z_{1}+r z_{2},\left(0 \leq r \leq 1, r=r^{*}\right) \text {, }
$$

with real parameter $r$. For the parameter value $r=\frac{1+z_{2}+z_{4}}{z_{2}}$ (numerically $r \approx 0.554958$ ) one finds that the value $z$ on the line between $z_{1}$ and $z_{1}+z_{2}$ coincides with the point $\frac{1+i \sqrt{7}}{2}$ according to

$$
\begin{gathered}
r=\frac{1+z_{2}+z_{4}}{z_{2}}=z_{2}^{-1}+1+z_{2}=z_{2}^{*}+1+z_{2}=r^{*}, \\
\Rightarrow z=z_{1}+\frac{1+z_{2}+z_{4}}{z_{2}} z_{2}=1+z_{1}+z_{2}+z_{4}=\frac{1+\mathrm{i} \sqrt{7}}{2} .
\end{gathered}
$$

Thus we have to bring the second arm of the absolute minimum 3-arm bicompasses in the position that it intersects the point $\left(\frac{1}{2}, \frac{\sqrt{7}}{2}\right)$ which last can 
be constructed by the intersection of the two mentioned circles and an alternative method of construction of the regular heptagon is described.

In addition, Figure 2 shows that the point $z=\frac{1+i \sqrt{7}}{2}$ lies also on the prolongation of the line between $z_{2}+z_{4}$ and $z_{2}$. This line can be parameterized with real parameter $s$ by

$$
z=z_{2}+s z_{4}, \quad\left(0 \leq s \leq 1, s=s^{*}\right) .
$$

For the parameter value $s=\frac{1+z_{1}+z_{4}}{z_{4}}$ (numerically $s \approx-0.801938$ ) one finds that the value $z$ on the prolongation of the line between $Z_{2}+z_{4}$ and $z_{2}$

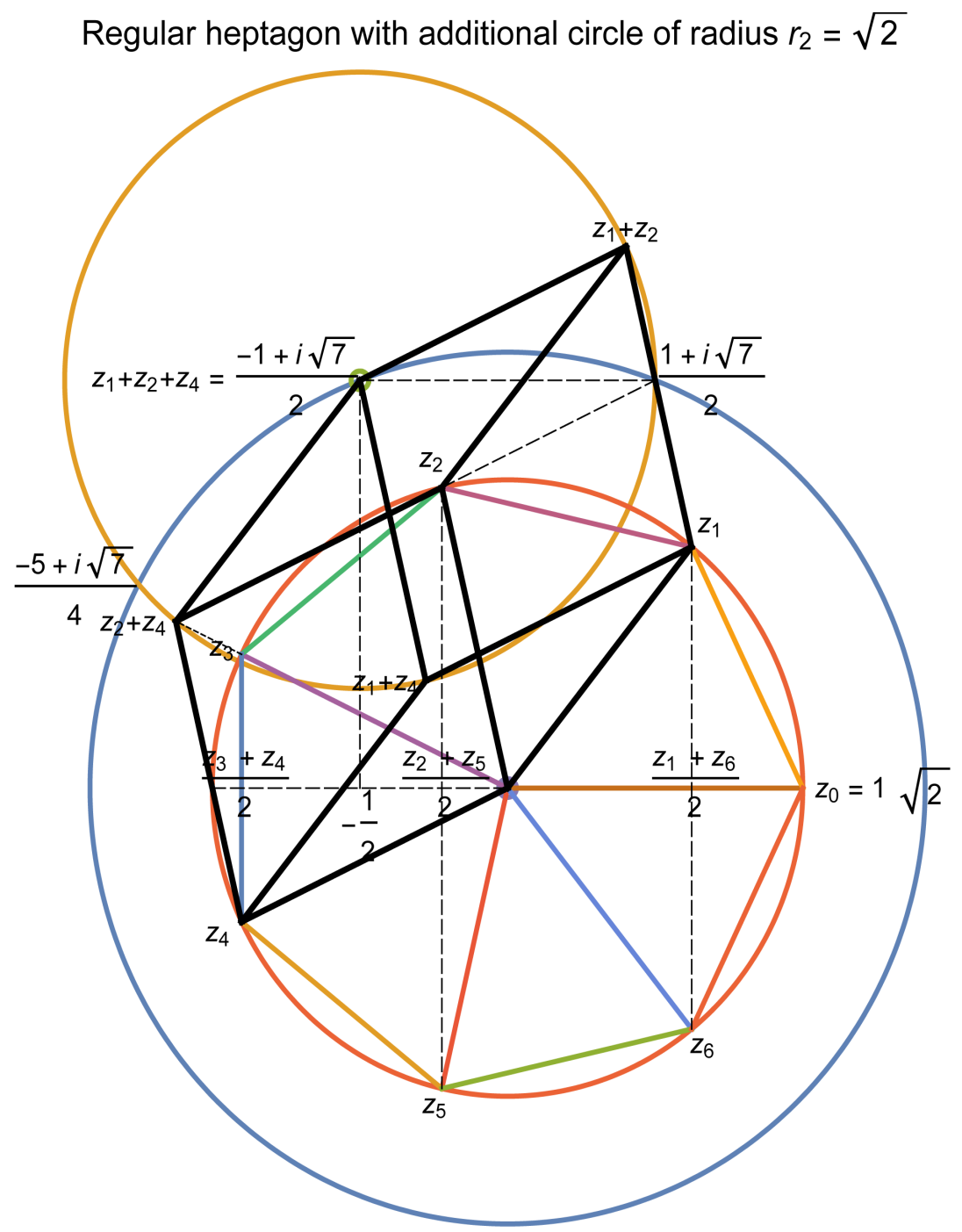

Figure 2. Regular heptagon and construction with rhombic bicompasses and ruler in complex $z$-plane. Additionally to the fixed points for the bicompasses and corresponding circles of radius $r_{1}=1$ we have drawn a circle around the coordinate origin with radius $r_{2}=\sqrt{2}$ and obtain in this way a modified construction by absolute minimum bicompasses with 3 arms (figure made as all following figures by "Mathematica 10"). 
coincides with the point $\frac{1+\mathrm{i} \sqrt{7}}{2}$ according to

$$
\begin{gathered}
s=\frac{1+z_{1}+z_{4}}{z_{4}}=z_{4}^{-1}+z_{4}+1=z_{4}^{*}+z_{4}+1=s^{*}, \\
\Rightarrow z=z_{2}+\frac{1+z_{1}+z_{4}}{z_{4}} z_{4}=1+z_{1}+z_{2}+z_{4}=\frac{1+\mathrm{i} \sqrt{7}}{2},
\end{gathered}
$$

that affirms the mentioned intersection.

In [1] it was already shown that the point $z=-1$ lies on the line between $z_{4}$ and $z_{2}+z_{4}$ which can be parameterized by

$$
z=z_{4}+t z_{2}, \quad\left(0 \leq t \leq 1, t=t^{*}\right),
$$

with real parameter $t$. With the parameter value $t=-\frac{1+z_{4}}{z_{2}}$ (numerically $t=0.445042)$ follows

$$
\begin{gathered}
t=-\frac{1+z_{4}}{z_{2}}=-\left(z_{2}^{*}+z_{2}\right)=t^{*}, \\
\Rightarrow z=z_{4}-\frac{1+z_{4}}{z_{2}} z_{2}=z_{4}-1-z_{4}=-1,
\end{gathered}
$$

that proves the statement. This, alternatively, can be also used for the construction of the regular heptagon by rhombic bicompasses and ruler. We mention that the parameters with the notation $r$ and $t$ are essentially the same since $r+t=1$.

The equation for the Cosines $u=z+z^{*}=2 x=2 \cos (\theta)$ (equation for $n=7$ in Table 1, Equation (2.4))

$$
\mathrm{U}_{3}\left(\frac{u}{2}\right)+\mathrm{U}_{2}\left(\frac{u}{2}\right)=u^{3}+u^{2}-2 u-1=0,
$$

is a 3-rd degree equation which cannot be solved only in quadratic radicals as it is known and its solution involves (complex) cubic radicals. Therefore, this does not help for the construction by compass and ruler. However, with other means of construction (e.g., neusis construction of angle trisection [2] [9] [14] and Gleason [13] (angle p-section)) this becomes possible.

Thus the regular heptagon loses a little its horror as not constructible by compass and ruler between the cases of the regular trigon $n=3$ and the regular octagon $n=8$ since it is constructible by bicompasses and ruler.

\section{Factorization of Cyclotomic Polynomial for $\boldsymbol{n}=9$ in Different Ways}

Since $n=9$ is a composite number we find different favorable factorizations of the cyclotomic polynomials. As special roots the circular division of the unit circle in 9 equal sectors contains the third roots of unity and we have the factorization 


$$
\begin{aligned}
\frac{z^{9}-1}{z-1} & =z^{8}+z^{7}+z^{6}+z^{5}+z^{4}+z^{3}+z^{2}+z+1 \\
& =\left(z^{2}+z+1\right)\left\{z^{6}+z^{3}+1\right\} \\
& =\left(z+\frac{1-i \sqrt{3}}{2}\right)\left(z+\frac{1+\mathrm{i} \sqrt{3}}{2}\right)\left\{\left(z^{3}+\frac{1-\mathrm{i} \sqrt{3}}{2}\right)\left(z^{3}+\frac{1+\mathrm{i} \sqrt{3}}{2}\right)\right\} \\
& =\left(z-z_{3}\right)\left(z-z_{6}\right)\left\{\left(\left(z-z_{1}\right)\left(z-z_{4}\right)\left(z-z_{7}\right)\right)\left(\left(z-z_{2}\right)\left(z-z_{5}\right)\left(z-z_{8}\right)\right)\right\},
\end{aligned}
$$

where the second factor in braces is of the form of the first factor with the substitution $z \rightarrow z^{3}$. It requires the angle trisections of the angles to the roots $z_{3}=z_{1}^{3}=\frac{-1+i \sqrt{3}}{2}$ and $z_{6}=z_{2}^{3}=\frac{-1-i \sqrt{3}}{2}=z_{3}^{*}$. This is possible by the neusis construction known from ancient time [2] [9] which, however, is not in the spirit of constructions by compass and ruler. The last is impossible for almost all arbitrary angles including the angle $\frac{2 \pi}{3}$. Therefore, as known, the circle division problem in case of $n=9$ cannot be solved by compass and ruler since the third root of an arbitrary complex number (here of $z_{3,6}=\frac{-1 \pm i \sqrt{3}}{2}$ ) cannot be constructed in this way. This can be also seen from the equation of Table 1 (Equation (2.4)) for the doubled Cosines $u_{k}=2 \cos \left(k \frac{2 \pi}{9}\right), \quad(k=1,2, \cdots, 8)$ as the solutions of the following polynomial equation in factorized form

$$
\mathrm{U}_{4}\left(\frac{u}{2}\right)+\mathrm{U}_{3}\left(\frac{u}{2}\right)=(u+1)\left(u^{3}-3 u+1\right)=0 .
$$

From the coefficients of the vanishing cubic polynomial follows

$$
\cos \left(\frac{2 \pi}{9}\right)+\cos \left(\frac{4 \pi}{9}\right)+\cos \left(\frac{8 \pi}{9}\right)=0, \quad \cos \left(\frac{2 \pi}{9}\right) \cos \left(\frac{4 \pi}{9}\right) \cos \left(\frac{8 \pi}{9}\right)=-\frac{1}{8}
$$

where the second relation does not provide independent in formation in comparison to the first. The cubic equation $u^{3}-3 u+1=0$ can be solved by cubic but not by quadratic radicals alone.

The 'standard' factorization into two polynomials of 4 -th degree is

$$
\begin{aligned}
\frac{z^{9}-1}{z-1} & =\left\{z^{4}+\frac{1-\mathrm{i} \sqrt{3}}{2} z^{3}+\frac{1+\mathrm{i} \sqrt{3}}{2} z+1\right\}\left\{z^{4}+\frac{1+\mathrm{i} \sqrt{3}}{2} z^{3}+\frac{1-\mathrm{i} \sqrt{3}}{2} z+1\right\} \\
& =\left\{\left(z-z_{6}\right)\left(\left(z-z_{1}\right)\left(z-z_{4}\right)\left(z-z_{7}\right)\right)\right\}\left\{\left(z-z_{3}\right)\left(\left(z-z_{2}\right)\left(z-z_{5}\right)\left(z-z_{8}\right)\right)\right\} .
\end{aligned}
$$

In the factor polynomials of 4 -th degree are contained two 3-cycles $\left(z_{1}, z_{4}, z_{7}\right)$ and $\left(z_{2}, z_{8}, z_{32} \equiv z_{5}\right)$ paired with one of the third roots $z_{3}$ and $z_{6}$ of unity. Other genuine than 3-and 6-cycles do not exist in case of $n=9$ but it happens that the root $z_{0}=1$ appears in the determination of the cycles according to the general procedure (it is then no more a cycle) that for prime $n$ is impossible.

A similar interestingly simple factorization by two polynomials of 4 -th degree follows directly from (7.1) by the product 


$$
\begin{aligned}
\frac{z^{9}-1}{z-1} & =\left\{z^{4}+\frac{1-\mathrm{i} \sqrt{3}}{2}\left(z^{3}+z\right)-\frac{1+\mathrm{i} \sqrt{3}}{2}\right\}\left\{z^{4}+\frac{1+\mathrm{i} \sqrt{3}}{2}\left(z^{3}+z\right)-\frac{1-\mathrm{i} \sqrt{3}}{2}\right\} \\
& =\left\{\left(z-z_{3}\right)\left(\left(z-z_{1}\right)\left(z-z_{4}\right)\left(z-z_{7}\right)\right)\right\}\left\{\left(z-z_{6}\right)\left(\left(z-z_{2}\right)\left(z-z_{5}\right)\left(z-z_{8}\right)\right)\right\} .
\end{aligned}
$$

and means the exchange of the factors $z-z_{3}$ and $z-z_{6}$. It is possible due to $n=9$ as a composite number.

\section{Factorization of Cyclotomic Polynomial for $n=11$ with Quadratic Radicals}

In case of $n=11$ one has only the following factorization by 5 -cycles which leads to polynomials with quadratic radicals in the coefficients

$$
\begin{aligned}
\frac{z^{11}-1}{z-1}= & z^{10}+z^{9}+z^{8}+z^{7}+z^{6}+z^{5}+z^{4}+z^{3}+z^{2}+z+1 \\
= & \left(z^{5}+\frac{1-i \sqrt{11}}{2} z^{4}-z^{3}+z^{2}-\frac{1+i \sqrt{11}}{2} z-1\right) \\
& \cdot\left(z^{5}+\frac{1+i \sqrt{11}}{2} z^{4}-z^{3}+z^{2}-\frac{1-i \sqrt{11}}{2} z-1\right) \\
= & \left(\left(z-z_{1}\right)\left(z-z_{3}\right)\left(z-z_{4}\right)\left(z-z_{5}\right)\left(z-z_{9}\right)\right) \\
& \cdot\left(\left(z-z_{2}\right)\left(z-z_{6}\right)\left(z-z_{7}\right)\left(z-z_{8}\right)\left(z-z_{10}\right)\right) .
\end{aligned}
$$

The 5-cycle in the first factor is formed by the roots $\left(z_{1}, z_{3}, z_{9}, z_{27}=z_{5}, z_{15}=z_{4},\left(z_{12}=z_{1}\right)\right)$ and the second factor by the complex conjugate roots $\left(z_{2}, z_{6}, z_{18}=z_{7}, z_{21}=z_{10}, z_{30}=z_{8},\left(z_{24}=z_{2}\right)\right)$, all $(\bmod 11)$.

The equation for the doubled Cosines $u=z+z^{*}=2 x=2 \cos (\theta)$ (case $n=11$ in Table 1 (Equation (2.4)) as a genuine 5-th order equation without special symmetries is not possible to solve in radicals as it is known.

\section{Factorizations of Cyclotomic Polynomial for $n=13$ with Quadratic Radicals}

The case $n=13$ is very interesting due to factorization of the cyclotomic equation by polynomials of 3-rd degree with only quadratic radicals in the coefficients in 3-cycles. This makes it possible for the application of the rhombic bicompasses and ruler for the construction of the regular tridecagon (13-gon).

The first factorization by two 6-cycles provides two factor polynomials of 6-th degree with real coefficients

$$
\begin{aligned}
\frac{z^{13}-1}{z-1}= & \sum_{k=0}^{12} z^{k}=\left\{z^{6}+\frac{1-\sqrt{13}}{2} z^{5}+2 z^{4}-\frac{1+\sqrt{13}}{2} z^{3}+2 z^{2}+\frac{1-\sqrt{13}}{2} z+1\right\} \\
& \cdot\left\{z^{6}+\frac{1+\sqrt{13}}{2} z^{5}+2 z^{4}-\frac{1-\sqrt{13}}{2} z^{3}+2 z^{2}+\frac{1+\sqrt{13}}{2} z+1\right\} \\
= & \left\{\left(z-z_{1}\right)\left(z-z_{3}\right)\left(z-z_{4}\right)\left(z-z_{9}\right)\left(z-z_{10}\right)\left(z-z_{12}\right)\right\} \\
& \cdot\left\{\left(z-z_{2}\right)\left(z-z_{5}\right)\left(z-z_{6}\right)\left(z-z_{7}\right)\left(z-z_{8}\right)\left(z-z_{11}\right)\right\} .
\end{aligned}
$$


Each of the two factor polynomials of 6-th degree can be again factorized in two polynomials of 3-rd degree with only quadratical radicals in the coefficients and the involved zeros we find from the four 3-cycles with the first involving the element $z_{1}$ explicitly given in (3.4)

$$
\begin{aligned}
\frac{z^{13}-1}{z-1}= & \left\{\left(z^{3}+\frac{1-\sqrt{13}-i \sqrt{2(13-3 \sqrt{13})}}{4} z^{2}-\frac{1-\sqrt{13}+i \sqrt{2(13-3 \sqrt{13})}}{4} z-1\right)\right. \\
& \left.\cdot\left(z^{3}+\frac{1-\sqrt{13}+i \sqrt{2(13-3 \sqrt{13})}}{4} z^{2}-\frac{1-\sqrt{13}-i \sqrt{2(13-3 \sqrt{13})}}{4} z-1\right)\right\} \\
& \cdot\left\{\left(z^{3}+\frac{1+\sqrt{13}-i \sqrt{2(13+3 \sqrt{13})}}{4} z^{2}-\frac{1+\sqrt{13}+i \sqrt{2(13+3 \sqrt{13})}}{4} z-1\right)\right. \\
& \left.\cdot\left(z^{3}+\frac{1+\sqrt{13}+i \sqrt{2(13+3 \sqrt{13})}}{4} z^{2}-\frac{1+\sqrt{13}-i \sqrt{2(13+3 \sqrt{13})}}{4} z-1\right)\right\} \\
= & \left\{\left(\left(z-z_{1}\right)\left(z-z_{3}\right)\left(z-z_{9}\right)\right)\left(\left(z-z_{4}\right)\left(z-z_{10}\right)\left(z-z_{12}\right)\right)\right\} \\
& \cdot\left\{\left(\left(z-z_{2}\right)\left(z-z_{5}\right)\left(z-z_{6}\right)\right)\left(\left(z-z_{7}\right)\left(z-z_{8}\right)\left(z-z_{11}\right)\right)\right\} .
\end{aligned}
$$

This means that the fixed points of the two rhombic bicompasses besides the coordinate origin $z=0$ are the following quadratic radicals $z=z_{1}+z_{3}+z_{9}$ and $z=z_{2}+z_{5}+z_{6}$ or their complex conjugates

$$
\begin{aligned}
z_{1}+z_{3}+z_{9} & =\frac{\sqrt{13}-1+i \sqrt{2(13-3 \sqrt{13})}}{4}=z_{12}^{*}+z_{10}^{*}+z_{4}^{*} \\
& \approx 0.651388+i 0.522416, \\
\left|z_{1}+z_{3}+z_{9}\right|^{2}= & \frac{5-\sqrt{13}}{2} \approx 0.697224, \quad\left|z_{1}+z_{3}+z_{9}\right| \approx 0.835000, \\
z_{2}+z_{5}+z_{6} & =\frac{-\sqrt{13}-1+i \sqrt{2(13+3 \sqrt{13})}}{4}=z_{11}^{*}+z_{8}^{*}+z_{9}^{*} \\
& \approx-1.15139+\mathrm{i} 1.72542, \\
\left|z_{2}+z_{5}+z_{6}\right|^{2} & =\frac{5+\sqrt{13}}{2} \approx 4.30278, \quad\left|z_{2}+z_{5}+z_{6}\right| \approx 2.07431 .
\end{aligned}
$$

Together with the circle division problem the two principally possible fixed points (without the complex conjugate ones) for the bicompasses are shown in Figure 3.

The sums and differences of the fixed points are also expressible by only quadratic radicals. We find for the sums algebraically and numerically

$$
\left(z_{1}+z_{3}+z_{9}\right)+\left(z_{2}+z_{5}+z_{6}\right)=\frac{-1+\mathrm{i} \sqrt{13+2 \sqrt{13}}}{2} \approx-0.5+\mathrm{i} 2.24784
$$



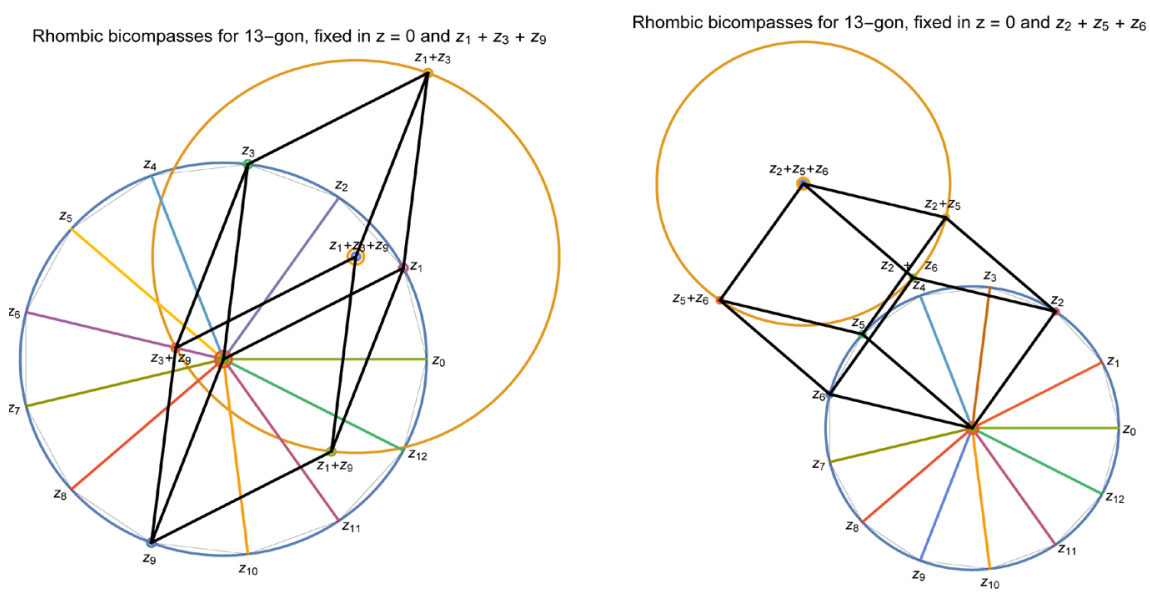

Figure 3. Two variants of rhombic bicompasses in case of a regular 13-gon. The two fixed points of the rhombic bicompasses are $z=0$ and $z=z_{1}+z_{3}+z_{9}$ in first picture and $z=0$ and $z=z_{2}+z_{5}+z_{6}$ in second picture (see text).

$$
\begin{gathered}
\left|\left(z_{1}+z_{3}+z_{9}\right)+\left(z_{2}+z_{5}+z_{6}\right)\right|=\frac{\sqrt{13}+1}{2} \approx 2.30278, \\
\left(z_{1}+z_{3}+z_{9}\right)+\left(z_{7}+z_{8}+z_{11}\right)=\frac{-1-\mathrm{i} \sqrt{13-2 \sqrt{13}}}{2} \approx-0.5-\mathrm{i} 1.20301, \\
\left|\left(z_{1}+z_{3}+z_{9}\right)+\left(z_{7}+z_{8}+z_{11}\right)\right|=\frac{\sqrt{13}-1}{2} \approx 1.30278,
\end{gathered}
$$

and for the differences

$$
\begin{gathered}
\left(z_{1}+z_{3}+z_{9}\right)-\left(z_{2}+z_{5}+z_{6}\right)=\frac{\sqrt{13}-\mathrm{i} \sqrt{13-2 \sqrt{13}}}{2} \approx 1.80278-\mathrm{i} 1.20301, \\
\left|\left(z_{1}+z_{3}+z_{9}\right)-\left(z_{2}+z_{5}+z_{6}\right)\right|=\sqrt{\frac{13-\sqrt{13}}{2}} \approx 2.16731, \\
\left(z_{1}+z_{3}+z_{9}\right)-\left(z_{7}+z_{8}+z_{11}\right)=\frac{\sqrt{13}+\mathrm{i} \sqrt{13+2 \sqrt{13}}}{2} \approx 1.80278+\mathrm{i} 2.24784, \\
\left|\left(z_{1}+z_{3}+z_{9}\right)-\left(z_{7}+z_{8}+z_{11}\right)\right|=\sqrt{\frac{13+\sqrt{13}}{2}} \approx 2.88145 .
\end{gathered}
$$

All these numbers and radiuses are constructible by compass and ruler. The points $z_{k},(k \neq 0 \bmod 13)$ alone and combinations of only two such points in form of $z_{k}+z_{l}$ are not constructible by compass and ruler since they contain cubic radicals.

This is illustrated in Figure 4 and Figure 5 where the two additional possibilities with the complex conjugate fixed points are obtained by reflection of each partial picture at the horizontal line through the coordinate origin. This provides 4 possibilities for the common combination of the two rhombic bicompasses where two of them are obtained again by reflection on the horizontal line through the coordinate origin. The two essentially different ones 
Two bicompasses for $13-$ gon with paired fixpoints $\left(0, z_{1}+z_{3}+z_{9}\right)$ and $\left(0, z_{2}+z_{5}+z_{6}\right)$

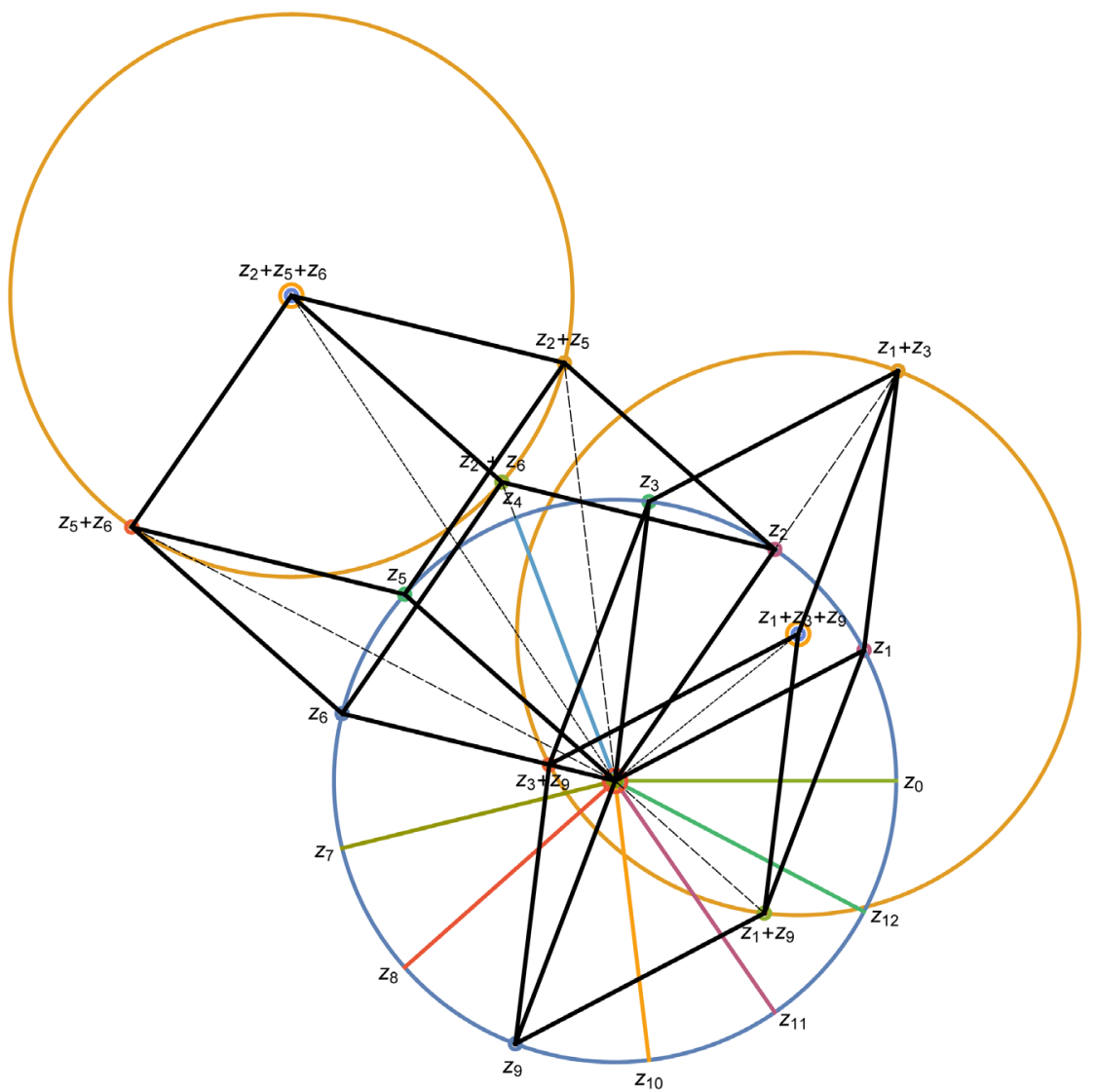

Figure 4. First of two variants of correlated rhombic bicompasses in case of a regular 13-gon.

are illustrated in Figure 4 and Figure 5.

According to Table 1 (Equation (2.4)) the doubled Cosines $u=2 \cos \left(k \frac{2 \pi}{13}\right)=2 \cos \left((13-k) \frac{2 \pi}{13}\right), \quad(k=1,2, \cdots, 6)$ are the roots of the 6 -th degree polynomial equation with 6 real-valued solutions

$$
\mathrm{U}_{6}\left(\frac{u}{2}\right)+\mathrm{U}_{5}\left(\frac{u}{2}\right)=u^{6}+u^{5}-5 u^{4}-4 u^{3}+6 u^{2}+3 u-1=0 .
$$

The polynomial on the left-hand side of this equation can be factorized in two polynomials of 3-rd degree with only quadratic radicals in the coefficients in the following way [13]

$$
\begin{aligned}
& u^{6}+u^{5}-5 u^{4}-4 u^{3}+6 u^{2}+3 u-1 \\
& =\left(u^{3}-\frac{\sqrt{13}-1}{2} u^{2}-u+\frac{\sqrt{13}-3}{2}\right)\left(u^{3}+\frac{\sqrt{13}+1}{2} u^{2}-u-\frac{\sqrt{13}+3}{2}\right) .
\end{aligned}
$$

This corresponds to the following relations for sums of the Cosines

$$
\cos \left(k \frac{2 \pi}{13}\right)=\cos \left((13-k) \frac{2 \pi}{13}\right)
$$




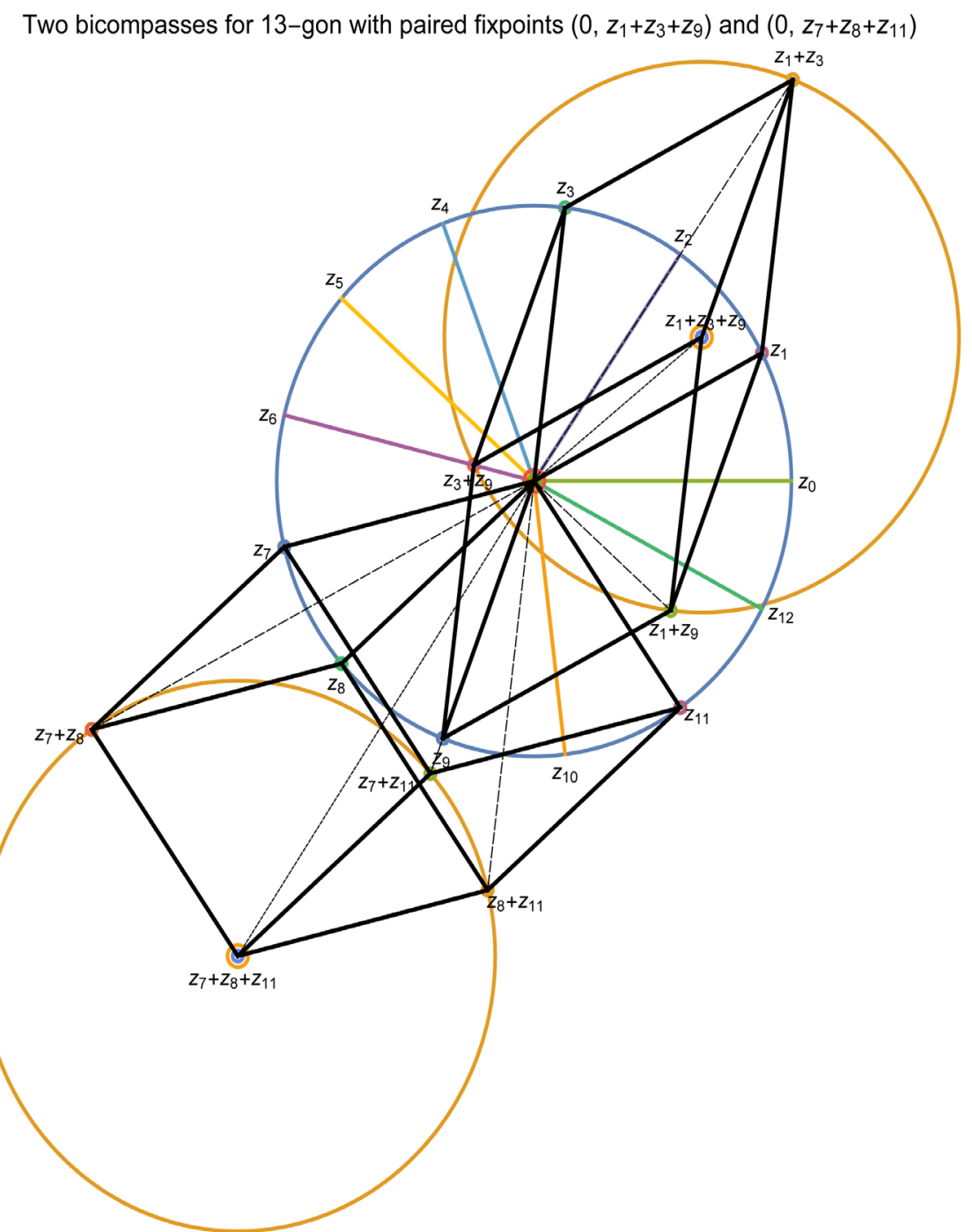

Figure 5. Second of two variants of correlated rhombic bicompasses in case of a regular 13-gon.

$$
\begin{gathered}
\cos \left(\frac{2 \pi}{13}\right)+\cos \left(\frac{6 \pi}{13}\right)+\cos \left(\frac{8 \pi}{13}\right)=\frac{\sqrt{13}-1}{4}, \\
\cos \left(\frac{4 \pi}{13}\right)+\cos \left(\frac{10 \pi}{13}\right)+\cos \left(\frac{12 \pi}{13}\right)=-\frac{\sqrt{13}+1}{4},
\end{gathered}
$$

and for products of the same Cosines

$$
\begin{aligned}
& \cos \left(\frac{2 \pi}{13}\right) \cos \left(\frac{6 \pi}{13}\right) \cos \left(\frac{8 \pi}{13}\right)=-\frac{\sqrt{13}-3}{16}, \\
& \cos \left(\frac{4 \pi}{13}\right) \cos \left(\frac{10 \pi}{13}\right) \cos \left(\frac{12 \pi}{13}\right)=\frac{\sqrt{13}+3}{16} .
\end{aligned}
$$

One may obtain these relations from relations (9.3) for the fixed points of the rhombic bicompasses. Relations (9.9) do not give additional independent information to relations (9.8). This can be seen if one transforms the products 
in (9.9) into sums using addition theorems for trigonometric functions. The same is true for the relations obtainable from the coefficients in front of $u$ in (9.7).

Equation (9.6) together with (9.7) means that the cyclotomic equation for $n=13$ can be exactly solved by not higher than cubic radicals. This, however, is not appropriate for the construction by compass and ruler which allows only quadratic radicals. The same conclusion can be drawn for the full solutions $z_{k}$ of the corner points of the regular 13-gon by setting equal to zero the four 3-rd degree polynomials in (9.2) of the factored cyclotomic polynomial (9.1).

The real construction of the regular 13-gon by rhombic bicompasses and ruler seems to be rather complicated. One may distinguish two principal cases:

1. The use of only one of the two possible bicompasses shown in the two pictures in Figure 3.

2. The use of the two rhombic bicompasses in correlated way in one of the two principally different variants as illustrated in Figure 4 and Figure 5.

The problem in first case is that during the application of the bicompasses, for example, to points $z_{1}$ and $z_{3}$ in first partial picture in Figure 3 one has to find the position when the angle between $z_{1}$ and $z_{3}$ is the doubled angle between $z_{0}=1$ and $z_{1}$ that is equivalent to solve the trisection of the angle between $z_{0}$ and $z_{3}$. The same problem arises if we use the points $z_{1}$ and $z_{9}$ or $z_{3}$ and $z_{9}$ instead of $z_{1}$ and $z_{3}$. In principal, an angle trisection can be made by a neusis construction attributed as already said to Archimedes [2] [9] but it is not in the spirit of construction by compass and ruler and, furthermore, it is unclear how it could be combined at the same time with the action of the bicompasses. We could not find a possibility also in case if we use in addition to one of the bicompasses the corresponding bicompasses with the complex conjugate fixed point.

The second case with correlated bicompasses seems to be, in principal, possible. In case of the regular heptagon we found points from the pictures on the arms of the bicompasses or on their prolongation which can be expressed by not higher than quadratic radicals and proved this property then algebraically. In case of the 13-gon we go a similar way but start from an opposite point of view. We look for possibilities of points on the lines between corners of bicompasses which can be represented by not higher than quadratic radicals. We consider the bicompasses which in the right position determine the corner points $\left(z_{1}, z_{3}, z_{9}\right)$ of the regular 13-gon (see Figure 4$)$. A first possibility is then to look on the line between $z_{1}$ and $z_{1}+z_{6}$ with $z_{6}$ on the other bicompasses which can be parameterized with real parameter $r$ by

$$
z=z_{1}+r z_{6}, \quad\left(r=r^{*}\right) .
$$

With the choice of a real parameter $r=\frac{z_{3}+z_{9}+\lambda z_{6}}{z_{6}}$ with real parameter $\lambda$ we find 


$$
\begin{gathered}
r=\frac{z_{3}+z_{9}+\lambda z_{6}}{z_{6}}=z_{3}^{*}+z_{3}+\lambda=r^{*}, \quad\left(\lambda=\lambda^{*}\right), \\
\Rightarrow z=z_{1}+\frac{z_{3}+z_{9}+\lambda z_{6}}{z_{6}} z_{6}=z_{1}+z_{3}+z_{9}+\lambda z_{6}=\frac{\sqrt{13}-1+i \sqrt{2(13-3 \sqrt{13})}}{4}+\lambda z_{6}, \\
\Rightarrow \lambda=0 .
\end{gathered}
$$

The points $\lambda z_{6}$ with real $\lambda \neq 0$ are, in general, and contrary to $z_{1}+z_{3}+z_{9}$ not representable by only quadratic radicals and, therefore, we set $\lambda=0$ in (9.11).

An analogous second possibility to the described one is to look on the line between $z_{3}$ of the first bicompasses and $z_{3}+z_{5}$ with $z_{5}$ of the second bicompasses which can be parameterized with real parameter $s$ by

$$
z=z_{3}+s z_{5}, \quad\left(s=s^{*}\right) .
$$

Then with the choice of parameter $s=\frac{z_{1}+z_{9}}{z_{5}}$ we find

$$
\begin{gathered}
s=\frac{z_{1}+z_{9}}{z_{5}}=z_{4}^{*}+z_{4}=s^{*}, \\
\Rightarrow z=z_{3}+\frac{z_{1}+z_{9}}{z_{5}} z_{5}=z_{3}+z_{1}+z_{9} .
\end{gathered}
$$

A third equivalent possibility is to look on the line between $z_{9}$ on the first bicompasses and $z_{9}+z_{2}$ with $z_{2}$ on the second bicompasses. It can be parameterized with real parameter $t$ by

$$
z=z_{9}+t z_{2}, \quad\left(t=t^{*}\right) .
$$

With the choice $t=\frac{z_{1}+z_{3}}{z_{2}}$ we obtain

$$
\begin{gathered}
t=\frac{z_{1}+z_{3}}{z_{2}}=z_{1}^{*}+z_{1}=t^{*}, \\
\Rightarrow z=z_{9}+\frac{z_{1}+z_{3}}{z_{2}} z_{2}=z_{9}+z_{1}+z_{3} .
\end{gathered}
$$

All three possibilities lead to the fixed point $z_{1}+z_{3}+z_{9}$ of the first bicompasses expressible by quadratic radical through which or through their prolongations the considered parameterized lines have to go. There are also further equivalent possibilities to use instead of the constructible points $z_{1}+z_{3}+z_{9}$ and $z_{2}+z_{5}+z_{6}$ their sums and differences which we did not investigate up to now in detail.

For the real construction one has to establish a correlation between the two bicompasses with fixed points $\left(0, z_{1}+z_{3}+z_{9}\right)$ and $\left(0, z_{2}+z_{5}+z_{6}\right)$ in such a way that when one of these bicompasses is in the right position the second at the same time has also to be in the right position for the angle division in thirteen equal parts. This means that we have to guarantee that the second of the bicompasses acts in concerted way with the first. It seems that one can use for 
such a coupling, for example, that the point $z_{3}+z_{9}$ of the first bicompasses lies exactly on the line (arm) between $z=0$ and $z_{6}$ of the second bicompasses (see Figure 4). This is already clear from symmetry and does not need to be proved. One has to guarantee that the point equivalent to $z_{3}+z_{9}$ in the right position of the bicompasses may glide along the line from $z=0$ to $z_{6}$ in the right position during the action of the two bicompasses. Then the point $z_{2}$ of the second bicompasses makes a bisection of the angle formed by the points $z_{1}$ and $z_{3}$ of the first bicompasses during their action when they arrive the right position. To determine this bisection seems to be possible during the action of the bicompasses. There are equivalent possibilities to realize the construction by the two bicompasses.

A weak point is the gliding of a point (here $z_{3}+z_{9}$ ) on a line (here between $z=0$ and $z_{6}$ ) as an admissible action in the spirit of geometric constructions from ancient time on. This is somehow problematic and requires more discussion in future as we are able to give here at this time.

There are also some not exact coincidences looking onto the figures one could think to be exact coincidences and which in the study of languages would be called 'false friends'. They may be used for approximate constructions of the 13-gon but it is not our intention to find and discuss them in detail. We mention only a few ones which are evident from the figures. The point $z_{12}$ lies on the circle with radius equal to 1 around the coordinate origin but not at the same time on the circle with radius equal to 1 around $z_{1}+z_{3}+z_{9}$ that is already optically to sense in Figure 4 and Figure 5 . The projection of the point $z_{1}+z_{3}$ onto the real $x$-axis is not exactly equal to 1 as it seems to be from Figure 4 and numerically we find $z_{1}+z_{3} \approx 1.005993+\mathrm{i} 1.457432$ where the deviation of the real part from 1 is widely above the numerical errors of calculation and it is nothing more to prove in this case. This is also clear since $z_{1}+z_{3}$ is not representable by quadratic radicals. The line from coordinate origin 0 to fixed point $z_{2}+z_{5}+z_{6}$ does not bisect the angle between $z_{4}$ and $z_{5}$ as from parameterized line set equal to the bisected angle $t\left(z_{2}+z_{5}+z_{6}\right)=\exp \left(i \frac{9 \pi}{13}\right)$

with numerically calculated complex (but not real) $t=0.482028+\mathrm{i} 0.00757137$ follows.

\section{Factorization of Cyclotomic Polynomial for $n=15$ with Quadratic Radicals}

According to the composite number $n=15$ the cyclotomic equation contains the cases of the cyclotomic equations for $n_{1}=3$ and $n_{2}=5$ as factors and possesses the form

$$
\begin{aligned}
\frac{z^{15}-1}{z-1}= & \sum_{k=0}^{14} z^{k}=\left(z^{2}+z+1\right)\left(z^{4}+z^{3}+z^{2}+z+1\right)\left(z^{8}-z^{7}+z^{5}-z^{4}+z^{3}-z+1\right) \\
= & \left(\left(z-z_{5}\right)\left(z-z_{10}\right)\right)\left(\left(z-z_{3}\right)\left(z-z_{6}\right)\left(z-z_{9}\right)\left(z-z_{12}\right)\right) \\
& \cdot\left(\left(z-z_{1}\right)\left(z-z_{2}\right)\left(z-z_{4}\right)\left(z-z_{7}\right)\left(z-z_{8}\right)\left(z-z_{11}\right)\left(z-z_{13}\right)\left(z-z_{14}\right)\right)
\end{aligned}
$$


The last factor is the irreducible part of the cyclotomic polynomial $p_{n}(z)$ (with coefficients in $\mathbb{Z}$ ) and is denoted by $\Phi_{15}(z)$ can be decomposed in two complex conjugate factors according to

$$
\begin{aligned}
\Phi_{15}(z)= & z^{8}-z^{7}+z^{5}-z^{4}+z^{3}-z+1 \\
= & \left(z^{4}-\frac{1+\mathrm{i} \sqrt{15}}{2} z^{3}-2 z^{2}-\frac{1-\mathrm{i} \sqrt{15}}{2} z+1\right) \\
& \cdot\left(z^{4}-\frac{1-\mathrm{i} \sqrt{15}}{2} z^{3}-2 z^{2}-\frac{1+\mathrm{i} \sqrt{15}}{2} z+1\right) \\
= & \left(\left(z-z_{1}\right)\left(z-z_{2}\right)\left(z-z_{4}\right)\left(z-z_{8}\right)\right) \\
& \cdot\left(\left(z-z_{7}\right)\left(z-z_{11}\right)\left(z-z_{13}\right)\left(z-z_{14}\right)\right) .
\end{aligned}
$$

In this factorizations we have two 4-cycles with the zeros $\left(z_{1}, z_{2}, z_{4}, z_{8}\right)$ and $\left(z_{14}, z_{13}, z_{11}, z_{7}\right)$ with $z_{1} z_{2} z_{4} z_{8}=z_{14} z_{13} z_{11} z_{7}=1$. From the given factorizations and from other considerations follow some known possibilities to solve the circle division problem for $n=15$ with compass and ruler and we do not further discuss this.

\section{Factorizations of Cyclotomic Polynomial for $\boldsymbol{n}=\mathbf{1 7}$ with Quadratic Radicals}

The possibility of the solution of the circle division problem in case $n=17$ together with the solution of the general problem from ancient time for which $n$ it can be solved at all by compass and ruler was discovered by the young Gauss and is represented in numerous books (e.g., [7] [9] [11] and others). The representation here of this known case illustrates our approach and facilitates its understanding in the unknown cases, in particular for $n=13$.

A first factorization of the cyclotomic polynomial which is palindromic in two palindromic polynomial factors with quadratic radicals in the coefficients is

$$
\begin{aligned}
\frac{z^{17}-1}{z-1}= & \sum_{k=0}^{16} z^{k} \\
= & \left\{\left(z^{8}+1\right)+\frac{1-\sqrt{17}}{2}\left(z^{7}+z\right)+\frac{5-\sqrt{17}}{2}\left(z^{6}+z^{2}\right)\right. \\
& \left.+\frac{7-\sqrt{17}}{2}\left(z^{5}+z^{3}\right)+(2-\sqrt{17}) z^{4}\right\} \\
& \cdot\left\{\left(z^{8}+1\right)+\frac{1+\sqrt{17}}{2}\left(z^{7}+z\right)+\frac{5+\sqrt{17}}{2}\left(z^{6}+z^{2}\right)\right. \\
& \left.+\frac{7+\sqrt{17}}{2}\left(z^{5}+z^{3}\right)+(2+\sqrt{17}) z^{4}\right\} \\
= & \left\{\left(z-z_{1}\right)\left(z-z_{2}\right)\left(z-z_{4}\right)\left(z-z_{8}\right)\left(z-z_{9}\right)\left(z-z_{13}\right)\left(z-z_{15}\right)\left(z-z_{16}\right)\right\} \\
& \cdot\left\{\left(z-z_{3}\right)\left(z-z_{5}\right)\left(z-z_{6}\right)\left(z-z_{7}\right)\left(z-z_{10}\right)\left(z-z_{11}\right)\left(z-z_{12}\right)\left(z-z_{14}\right)\right\} .
\end{aligned}
$$

The first factor, for example, involves the zeros 
$z_{1}, z_{2}, z_{4}, z_{8}, z_{16}, z_{32} \equiv z_{15}, z_{64} \equiv z_{30} \equiv z_{13}$ modulo 17 . The two palindromic factor polynomials of 8-th degree can be decomposed each in the product of two polynomials of 4-th degree which are also palindromic in the following way: first factor

$$
\begin{aligned}
& \left(z^{8}+1\right)+\frac{1-\sqrt{17}}{2}\left(z^{7}+z\right)+\frac{5-\sqrt{17}}{2}\left(z^{6}+z^{2}\right)+\frac{7-\sqrt{17}}{2}\left(z^{5}+z^{3}\right)+(2-\sqrt{17}) z^{4} \\
= & \left(\left(z^{4}+1\right)+\frac{1-\sqrt{17}-\sqrt{2(17-\sqrt{17})}}{4}\left(z^{3}+z\right)+\frac{7-\sqrt{17}+\sqrt{2(17+\sqrt{17})}}{4} z^{2}\right) \\
& \cdot\left(\left(z^{4}+1\right)+\frac{1-\sqrt{17}+\sqrt{2(17-\sqrt{17})}}{4}\left(z^{3}+z\right)+\frac{7-\sqrt{17}-\sqrt{2(17+\sqrt{17})}}{4} z^{2}\right) \\
= & \left(\left(z-z_{1}\right)\left(z-z_{4}\right)\left(z-z_{13}\right)\left(z-z_{16}\right)\right)\left(\left(z-z_{2}\right)\left(z-z_{8}\right)\left(z-z_{9}\right)\left(z-z_{15}\right)\right),
\end{aligned}
$$

second factor

$$
\begin{aligned}
& \left(z^{8}+1\right)+\frac{1+\sqrt{17}}{2}\left(z^{7}+z\right)+\frac{5+\sqrt{17}}{2}\left(z^{6}+z^{2}\right)+\frac{7+\sqrt{17}}{2}\left(z^{5}+z^{3}\right)+(2+\sqrt{17}) z^{4} \\
= & \left(\left(z^{4}+1\right)+\frac{1+\sqrt{17}-\sqrt{2(17+\sqrt{17})}}{4}\left(z^{3}+z\right)+\frac{7+\sqrt{17}-\sqrt{2(17-\sqrt{17})}}{4} z^{2}\right) \\
& \cdot\left(\left(z^{4}+1\right)+\frac{1+\sqrt{17}+\sqrt{2(17+\sqrt{17})}}{4}\left(z^{3}+z\right)+\frac{7+\sqrt{17}+\sqrt{2(17-\sqrt{17})}}{4} z^{2}\right) \\
= & \left(\left(z-z_{3}\right)\left(z-z_{5}\right)\left(z-z_{12}\right)\left(z-z_{14}\right)\right)\left(\left(z-z_{6}\right)\left(z-z_{7}\right)\left(z-z_{10}\right)\left(z-z_{11}\right)\right) .
\end{aligned}
$$

In the factorization we have a product of 4 palindromic polynomials of 4 -th degree each of which can be decomposed again in a product of 2 palindromic polynomials of now 2-nd degree with only quadratic radicals in the coefficients according to the general formula ${ }^{3}$

$$
\begin{aligned}
z^{4}+a z^{3}+b z^{2}+a z+1= & \left(z^{2}+\frac{a+\sqrt{a^{2}-4 b+8}}{2} z+1\right) \\
& \cdot\left(z^{2}+\frac{a-\sqrt{a^{2}-4 b+8}}{2} z+1\right) .
\end{aligned}
$$

This provides the further splitting in products of pairs of two palindromic polynomials of 2-nd degree as follows:

first factor

\footnotetext{
${ }^{3} \mathrm{~A}$ palindromic polynomial of 8-th degree cannot be decomposed, in general, in a product of 2 palindromic polynomials of 4-th degree with not higher than quadratic radicals in the coefficients such as in formulae (11.2) and (11.3) and this is only possible under certain restrictions to the coefficients of the 8-th degree palindromic polynomial. There are, however, also some interesting relatives to palindromic polynomials of $n$-th degree without special names. These are, in particular, polynomials where the coefficients in front of $z^{n-k}$ are equal to $( \pm 1)^{k}$ to that of $z^{k}$ or where they are complex conjugate to the coefficients in front of $z^{k}$ with possible additional factors $( \pm 1)^{k}$.
} 


$$
\begin{aligned}
& \left(z-z_{1}\right)\left(z-z_{4}\right)\left(z-z_{13}\right)\left(z-z_{16}\right)=\left(\left(z-z_{1}\right)\left(z-z_{16}\right)\right)\left(\left(z-z_{4}\right)\left(z-z_{13}\right)\right) \\
& =\left(z^{2}+\frac{1-\sqrt{17}-\sqrt{2(17-\sqrt{17})}-2 \sqrt{17+3 \sqrt{17}-\sqrt{2(85+19 \sqrt{17})}}}{8} z+1\right) \\
& \left(z^{2}+\frac{1-\sqrt{17}-\sqrt{2(17-\sqrt{17})}+2 \sqrt{17+3 \sqrt{17}-\sqrt{2(85+19 \sqrt{17})}}}{8} z+1\right)
\end{aligned}
$$

second factor

$$
\begin{aligned}
& \left(z-z_{2}\right)\left(z-z_{8}\right)\left(z-z_{9}\right)\left(z-z_{15}\right)=\left(\left(z-z_{2}\right)\left(z-z_{15}\right)\right)\left(\left(z-z_{8}\right)\left(z-z_{9}\right)\right)
\end{aligned}
$$

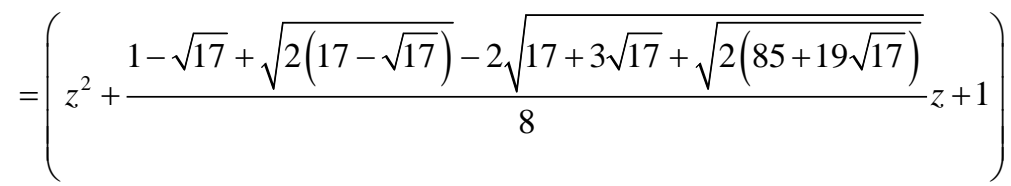

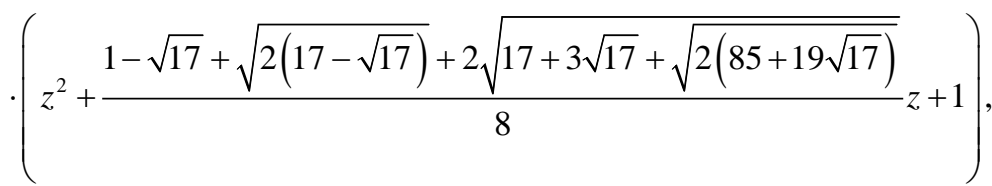

third factor

$$
\begin{aligned}
& \left(z-z_{3}\right)\left(z-z_{5}\right)\left(z-z_{12}\right)\left(z-z_{14}\right)=\left(\left(z-z_{3}\right)\left(z-z_{14}\right)\right)\left(\left(z-z_{5}\right)\left(z-z_{12}\right)\right)
\end{aligned}
$$

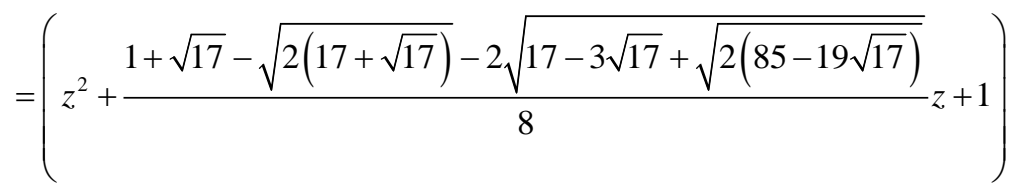

$$
\begin{aligned}
& \cdot\left(z^{2}+\frac{1+\sqrt{17}-\sqrt{2(17+\sqrt{17})}+2 \sqrt{17-3 \sqrt{17}+\sqrt{2(85-19 \sqrt{17})}}}{8} z+1\right),
\end{aligned}
$$

fourth factor

$$
\begin{aligned}
& \left(z-z_{6}\right)\left(z-z_{7}\right)\left(z-z_{10}\right)\left(z-z_{11}\right)=\left(\left(z-z_{6}\right)\left(z-z_{11}\right)\right)\left(\left(z-z_{7}\right)\left(z-z_{10}\right)\right) \\
& =\left(z^{2}+\frac{1+\sqrt{17}+\sqrt{2(17+\sqrt{17})}-2 \sqrt{17-3 \sqrt{17}-\sqrt{2(85-19 \sqrt{17})}}}{8} z+1\right)
\end{aligned}
$$

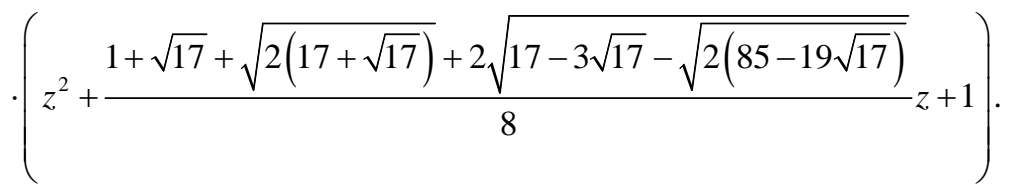

The bigger brackets in the short and in the explicit expressions correspond to each other in their ordering in the written form of the formulae.

The coefficients in all the polynomials of second degree in (11.5)-(11.8) in front of $z$ are real ones and the half of the negatively taken value provides the 
Cosine of the corresponding angle. From first factor in first polynomial in (11.5), for example, one find $\cos \left(\frac{2 \pi}{17}\right)$, that means the Cosine of the angle to the first corner of the 17-gon which is explicitly

$$
\cos \left(\frac{2 \pi}{17}\right)=\frac{-1+\sqrt{17}+\sqrt{2(17-\sqrt{17})}+2 \sqrt{17+3 \sqrt{17}-\sqrt{2(85+19 \sqrt{17})}}}{16} .
$$

This is identical in content (but not in form) with the expression derived by Gauss in Section 365 of Disquisitiones Arithmetica as given in the citation by Edwards [6] on p. 32 and here cited according to him (see also Stewart [5] (p. $232))$. All values for $\cos \left(k \frac{2 \pi}{17}\right),(k=1,2, \cdots, 8)$ can be taken from the corresponding coefficients in the quadratic factor polynomials in (11.5)-(11.8).

Let us give for completeness and for the factorization of the polynomial $\mathrm{U}_{8}\left(\frac{u}{2}\right)+\mathrm{U}_{7}\left(\frac{u}{2}\right)$ for $n=17$ in Table 1 (Equation (11.4)) with coefficients in form of quadratic radicals the complete expressions for the doubled Cosines for all primitive roots $u_{k}$. From (11.5) and (11.6) follows for

$$
\begin{gathered}
u_{k} \equiv 2 \cos \left(k \frac{2 \pi}{17}\right)=2 \cos \left((17-k) \frac{2 \pi}{17}\right) \\
u_{1,4}=\frac{-1+\sqrt{17}+\sqrt{2(17-\sqrt{17})} \pm 2 \sqrt{17+3 \sqrt{17}-\sqrt{2(85+19 \sqrt{17})}}}{8}, \\
u_{2,8}=\frac{-1+\sqrt{17}-\sqrt{2(17-\sqrt{17})} \pm 2 \sqrt{17+3 \sqrt{17}+\sqrt{2(85+19 \sqrt{17})}}}{8},
\end{gathered}
$$

where the first of the two indices in $u_{k, l}$ corresponds to the upper sign and the second to the lower one in " \pm " and, analogously, from (11.7) and (11.8)

$$
\begin{aligned}
& u_{3,5}=\frac{-1-\sqrt{17}+\sqrt{2(17+\sqrt{17})} \pm 2 \sqrt{17-3 \sqrt{17}+\sqrt{2(85-19 \sqrt{17})}}}{8}, \\
& u_{6,7}=\frac{-1-\sqrt{17}-\sqrt{2(17+\sqrt{17})} \pm 2 \sqrt{17-3 \sqrt{17}-\sqrt{2(85-19 \sqrt{17})}}}{8} .
\end{aligned}
$$

The factorization of $\sum_{l=0}^{16} z^{l}$ taken together with (11.5)-(11.8) can be formally written

$$
\begin{aligned}
\frac{z^{17}-1}{z-1} & =\sum_{l=0}^{16} z^{l}=\prod_{k=1}^{8}\left(z^{2}-u_{k} z+1\right), \\
u_{k} & \equiv 2 \cos \left(k \frac{2 \pi}{17}\right), \quad(k \in \mathbb{Z}(\bmod 17)),
\end{aligned}
$$

where the explicit form of the coefficients $u_{k},(k=1,2, \cdots, m=8)$ given in (11.10) and (11.11) are taken from (11.5) and (11.8). If we insert the expressions $u_{k}$ for $u$ into the cyclotomic Equation (2.5) for $m=8, n=2 m+1=17$ using Table 1 
(Equation (2.4))

$$
\begin{aligned}
\mathrm{U}_{8}\left(\frac{u}{2}\right)+\mathrm{U}_{7}\left(\frac{u}{2}\right) & =u^{8}+u^{7}-7 u^{6}-6 u^{5}+15 u^{4}+10 u^{3}-10 u^{2}-4 u+1 \\
& =\frac{p_{17}(z)}{(z-1) z^{8}}, \quad\left(u \equiv z+z^{-1}=z+z^{*}\right),
\end{aligned}
$$

then its vanishing is identically satisfied. We checked this algebraically by computer with "Mathematica" 4 and we checked also numerically by computer that the order of the braces and big brackets in different parts of the Formulae (11.2) and (11.3) and in Formulae (11.5)-(11.8) remains preserved.

Since in case of the regular 17-gon all Cosines of the angles are expressed by quadratic radicals the multiplication of arbitrary factors $\left(u-u_{k}\right)\left(u-u_{l}\right)$ leads again to polynomials with only quadratic radicals in the coefficients. Therefore, the factorization of cyclotomic polynomials such as (11.13) with quadratic radicals in the coefficients admits many possibilities but only a few lead to simple expressions. In a first step one may obtain the following factorization of the polynomial of 8-th degree on the right-hand side of (11.13) into the product of two polynomials of 4 -th degree with quadratic radicals as coefficients

$$
\begin{aligned}
& u^{8}+u^{7}-7 u^{6}-6 u^{5}+15 u^{4}+10 u^{3}-10 u^{2}-4 u+1 \\
&=\left(u^{4}+\frac{1-\sqrt{17}}{2} u^{3}-\frac{3+\sqrt{17}}{2} u^{2}+(2+\sqrt{17}) u-1\right) \\
&\left(u^{4}+\frac{1+\sqrt{17}}{2} u^{3}-\frac{3-\sqrt{17}}{2} u^{2}+(2-\sqrt{17}) u-1\right) .
\end{aligned}
$$

This is certainly the simplest of the possible factorizations of the 8-th degree polynomial in two polynomials with quadratic radicals as coefficients. The first factor polynomial contains the factors $\left(u-u_{1}, u-u_{4}, u-u_{2}, u-u_{8}\right)$ and the second the remaining factors $\left(u-u_{3}, u-u_{5}, u-u_{6}, u-u_{7}\right)$. Further factorizations of the polynomials of 4 -th degree into products of polynomials of 2-nd degree with quadratic radicals in the coefficients are to obtain in analogous way using Formulae (11.2)-(11.3) and since each factorization in factors $u-u_{k}$ contains only quadratic radicals this is even possible in different ways. We do not write down all this.

The general possibilities for the construction of regular n-gons by compass and ruler according to the rules of ancient time which young Gauss finally found and proved and which the problem solved forever are the following (e.g., [7] [9]). The basis form such Fermat numbers $F_{l} \equiv 2^{2^{l}}+1$ which are prime numbers $p_{i}$. We denote them by $p_{1}, p_{2}, \cdots$. An $n$-gon is constructible by compass and ruler if it is a power $2^{k}$ (for angle bisections) multiplied by an arbitrary product of distinct prime Fermat numbers $p_{i}$ that means

$$
n=2^{k} p_{i_{1}} p_{i_{2}} \cdots
$$

${ }^{4}$ The used program "Mathematica 10" does not automatically provide the solutions of (11.13) in the explicit form of the $u_{k}$ but affirms immediately the satisfaction of the equation if we insert there separately their explicit values. 
As it is well known the first 5 Fermat numbers which are $F_{0}=3, F_{1}=5, F_{2}=17, F_{3}=257, F_{4}=65537$ and are all prime numbers but the next Fermat number $F_{5}$ is composite (e.g., [7] [9]; see also Table 2 in Section 3). The analogous case of numbers $G_{l}=3 \cdot 2^{2^{l}}+1$ for geometric constructions by bicompasses and ruler are given also in Table 2 .

By combinations of constructions by compass and ruler with constructions by bicompasses and ruler one finds further possibilities to solve the circle division problem geometrically. For example, by combination of the constructions of the angles $\frac{2 \pi}{3}$ or $\frac{2 \pi}{5}$ with the angles $\frac{2 \pi}{7}$ or $\frac{2 \pi}{13}$ by bicompasses one may construct

$$
\begin{gathered}
\frac{2 \pi}{3} \mp \frac{2 \pi}{7}=\frac{(7 \mp 3) 2 \pi}{21}=\left\{\begin{array}{l}
2^{2} \frac{2 \pi}{21} \\
10 \frac{2 \pi}{21}
\end{array}\right\}, \quad \frac{2 \pi}{5} \mp \frac{2 \pi}{7}=\frac{(7 \mp 5) 2 \pi}{35}=\left\{\begin{array}{c}
2^{2} \frac{2 \pi}{35} \\
12 \frac{2 \pi}{35}
\end{array}\right\}, \\
\frac{2 \pi}{3} \mp \frac{2 \pi}{13}=\frac{(13 \mp 3) 2 \pi}{39}=\left\{\begin{array}{l}
10 \frac{2 \pi}{39} \\
2^{4} \frac{2 \pi}{39}
\end{array}\right\}, \quad \frac{2 \pi}{5} \mp \frac{2 \pi}{13}=\frac{(13 \mp 5) 2 \pi}{65}=\left\{\begin{array}{c}
2^{3} \frac{2 \pi}{65} \\
18 \frac{2 \pi}{35}
\end{array}\right\} .
\end{gathered}
$$

We see that in connection with (repeated) angle bisections admitted by powers of 2 in front of $\frac{2 \pi}{n}$ the circle division problem can be principally also solved, for example, for $n=21,35,39,65$. More generally, if the angles $\frac{2 \pi}{m}$ and $\frac{2 \pi}{n}$ are constructible, one may consider the combinations $k \frac{2 \pi}{m}+l \frac{2 \pi}{n}$ with $k \bmod m$ and $l \bmod n$ in analogous way.

\section{Is Factorization of Cyclotomic Polynomial for $n=19$ with 3-Cycles and with Only Quadratic Radicals Possible?}

In case of $n=2 m+1=19$ we find as a first factorization of the cyclotomic polynomial

$$
\begin{aligned}
\frac{z^{19}-1}{z-1}= & \sum_{k=0}^{18} z^{k}=\left\{z^{9}+\frac{1-\mathrm{i} \sqrt{19}}{2} z^{8}-2 z^{7}+\frac{3+\mathrm{i} \sqrt{19}}{2} z^{6}+\frac{5-\mathrm{i} \sqrt{19}}{2} z^{5}\right. \\
& \left.-\frac{5+\mathrm{i} \sqrt{19}}{2} z^{4}-\frac{3-\mathrm{i} \sqrt{19}}{2} z^{3}+2 z^{2}-\frac{1+\mathrm{i} \sqrt{19}}{2} z-1\right\} \\
& \cdot\left\{z^{9}+\frac{1+\mathrm{i} \sqrt{19}}{2} z^{8}-2 z^{7}+\frac{3-\mathrm{i} \sqrt{19}}{2} z^{6}+\frac{5+\mathrm{i} \sqrt{19}}{2} z^{5}\right. \\
& \left.-\frac{5-\mathrm{i} \sqrt{19}}{2} z^{4}-\frac{3+\mathrm{i} \sqrt{19}}{2} z^{3}+2 z^{2}-\frac{1-\mathrm{i} \sqrt{19}}{2} z-1\right\} \\
= & \left\{\left(z-z_{1}\right)\left(z-z_{4}\right)\left(z-z_{5}\right)\left(z-z_{6}\right)\left(z-z_{7}\right)\left(z-z_{9}\right)\left(z-z_{11}\right)\left(z-z_{16}\right)\left(z-z_{17}\right)\right\} \\
& \cdot\left\{\left(z-z_{2}\right)\left(z-z_{3}\right)\left(z-z_{8}\right)\left(z-z_{10}\right)\left(z-z_{12}\right)\left(z-z_{13}\right)\left(z-z_{14}\right)\left(z-z_{15}\right)\left(z-z_{18}\right)\right\}
\end{aligned}
$$


A further factorization of the two polynomial factors in three 3-cycles each with only quadratic radicals in the coefficients is not possible since such an ansatz leads to cubic equations for the coefficients. Therefore the construction of the 19-gon is not possible by compass and ruler as well as it is not possible by rhombic bicompasses and ruler.

\section{Some General Rules for the Factorization of the Special Palindromic Polynomials $\sum_{k=0}^{2 m} z^{2 m-k}$ in Two Factors with Quadratic Radicals in the Coefficients}

If one looks through the first factorizations of the cyclotomic polynomials $\frac{p_{2 m+1}(z)}{z-1}=\sum_{k=0}^{2 m} z^{2 m-k}, \quad(m=0,1,2, \cdots)$ for odd $n=2 m+1$ into the product of two 'similar' polynomials of $m$-th degree with only quadratic radicals in their coefficients as explicitly given up to $n=19, m=9$ one finds at once some regularities. First, one may distinguish two categories for odd $n$ first where the coefficients in the both factor polynomials possess real coefficients and second where they possess complex conjugate coefficients. It depends on the two cycles of length $m$ and on their content of roots. If each such factor involves together with each root also the conjugate root modulo $n$ then all coefficients are real ones. This is the case up to $n=19$ only for $n=5$ and $n=17$ which are prime Fermat numbers and for $n=13$. In all other cases up to $n=19$ as one can see from the given explicit factorization the coefficients in the two factor polynomials are complex conjugate to each other. One may take this into account if one knows the involved roots of the two cycles which lead to this factorization. From the coefficients in front of $z^{n-2}$ and $z^{n-3}$ of the cyclotomic polynomial $\frac{p_{n}(z)}{z-1}$ which are equal to 1 as for all its coefficients one can determine both coefficients $a_{m-1}$ and $a_{m-2}$ in the two factors of their principal form $z^{m}-a_{m-1} z^{m-1}+a_{m-2} z^{m-2}+\cdots+(-1)^{m}$. One may begin to determine the coefficients in the factorized form also from the low-order powers of $z$ or better at once from both sides, from the high-order and from the low-order powers of $z$. From the consequence (3.6) of the Fermat theorem follows, at least, for prime numbers $n=2 m+1$ that in the first factorization of the cyclotomic polynomial $\frac{p_{2 m+1}(z)}{z-1}$ into a product of two polynomials of degree $m$ with quadratic radicals only that the coefficients of the powers to $z^{0}=1$ can also be only \pm 1 depending on $m$ as an even or odd number. In next steps one has to look what follows from the symmetries and have to introduce unknown quantities for the coefficient which can be successively determined from the multiplication of the two factorized polynomials which have to give the initial cyclotomic polynomials. It is obvious how in the second-highest and second-lowest coefficients appear the square roots $\sqrt{n}$ of $n$ (for prime $n$ ) but for coefficients more to the middle of the polynomials it is difficult to derive general formulae. 


\section{The Number $\frac{-1+i \sqrt{7}}{2}$ as the Second of the Basis \\ Vectors of a Klein Lattice in the Complex Plane with Unique Prime Factorization}

We make now a small digression. Conway and Smith [21] describe from p. 15 on the lattices in the complex plane with respect to the factorization into products of prime complex lattice vectors. The unique factorization of Gaussian integers and also of Eisenstein integers can be found in number-theoretic works (e.g., [8]) but all possible lattices where such a unique factorization into products of prime factors is possible was up to now seldom to find. According to Conway and Smith [21] there are only 9 such lattices (up to rotations and scalings) and the proof for the completeness of these lattices with such a property goes back to Heegner and independently to Baker and Stark. What is very interesting for us although not directly connected with the unique prime factorization is that next to the Gaussian integers (possible basis $(1, \mathrm{i})$ and the Eisenstein integers (possible basis $\left(1, \frac{-1+\mathrm{i} \sqrt{3}}{2}\right)$ the Klein integers ('Kleinian ring') with possible basis $\left(1, \frac{-1+i \sqrt{7}}{2}\right)$ possesses this property [21]. The complex number $\frac{-1+i \sqrt{7}}{2}$ plays a main role as second of the fixed points besides the coordinate origin in our construction of the regular heptagon by rhombic bicompasses and ruler (see Section 6). The Klein lattice is formed by the set $z=z_{m, n}$ of all lattice points $z \in \mathbb{C}[21]$

$$
z_{m, n}=m+n\left(\frac{-1+\mathrm{i} \sqrt{7}}{2}\right), \quad(m, n=0, \pm 1, \pm 2, \cdots) .
$$

The norm of the lattice points $z_{m, n}$ that is the squared modulus $N(z)=|z|^{2}$ of an arbitrary complex number $z$ is

$$
N\left(z_{m, n}\right)=\left|m-\frac{n}{2}+\mathrm{in} \frac{\sqrt{7}}{2}\right|^{2}=m^{2}-m n+2 n^{2} \geq 0,
$$

which is a non-negative integer.

Similar pictures as they are known for the distribution of the Gauss primes and for the Eisenstein primes in the complex plane (see, e.g., Conway and Smith [9], p. 224 (in English Ed.) and [21], p. 18 and particularly beautiful (larger) in the book of Guy [22], pp. 56, 57) would be also very interesting for the Klein primes (with 7-fold symmetry $C_{7 v}$ as subgroup of permutation group $S_{7}$ ).

\section{Conclusions}

Present article results mainly from investigations to the problem for which $n$ a regular $n$-gon besides the regular heptagon $(n=7)$ may be constructed by 
rhombic bicompasses and ruler. We found that the next possible case is the regular tridecagon $(n=13)$ although some problems of the realization of the construction remained open. The technical design of rhombic bicompasses, in particular, if the arm length should be variable within certain limits, we do not consider as our problem to which we may contribute. We posed our mathematical problem into the general frame of the solution of the cyclotomic equation and of the factorization of the cyclotomic polynomials for arbitrary $n$ where only concerning the factorization with integer or rational coefficients (in $\mathbb{Z}$ or $\mathbb{Q}$ ) of the (irreducible) factor polynomials exists a well-known theory. It is a special case of the Galois theory of the solvability of polynomial equations in radicals. Our approach to the factorization in case of $n=13$ and in the other cases with odd $n$ to get factorization with coefficients which do not contain higher than quadratic radicals or cannot be resolved at all by radicals is similar to the usual approach in case of the 17-gon. The first task was to find the different cycles that is illustrated in detail for the cases $n=7$ and $n=13$. The factorization with only quadratic radicals in the coefficients is then straightforward using known details for the coefficients and determining the unknown from the general restrictions for the coefficients in systematic way.

Our bicompasses underlie hard restriction since they possess equal arm lengths and bundles of (maximum) three arms are connected with the two fixed points to guarantee to draw at once two circles of equal radius with correlation of points on the circles with one degree of freedom. The question is whether or not it is possible to solve other geometric construction problems with them as the described ones. However, the trisection of an arbitrary angle which is not possible with compass and ruler seems to be also not possible by bicompasses and ruler. It is due to the form of the equation for the Cosines of the trisected angle which is of third degree with vanishing coefficient in front of the 2-nd degree power which determines the two fixed points. The fixed points coincide in this case and the motion with one degree of freedom of the bicompasses is the rotation of a regular hexagon around the only fixed point. One position of this hexagon determines with 3 of its corner points the right position for the trisection of the angle but it cannot be determined which of the positions is the right. The restrictions to the bicompasses can be weakened. For example, the arm lengths can be varied and the number of arms between the fixed points may be increased to increase the degree of freedom. All this is a wide field and we are maybe only at the beginning of it.

In my previous article [1] I made some remarks about rare biological objects with 7-fold symmetry in heavy contrast to ubiquitous 5 -fold symmetry in many plant families (please, amend there the scientific name for oleander into Nerium oleander). In the meanwhile I looked through the book of Ernst Haeckel Kunstformen der Natur from (1899-1904) ${ }^{5}$ with the wonderful drawings and

${ }^{5}$ Edited by O. Breidbach with inclusion of some earlier publications of Ernst Haeckel and biographical notes under the title Kunstformen der Natur, Kunstformen aus dem Meer, Prestel-Verlag, 3. Auflage, München 2016. 
with the aim to see whether or not are there biological objects with 7-fold symmetry and found two such objects: 1. table 34 (object 2) a colony forming alga from genus Pediastrum of the green algae Chlorophyta of kingdom Protista (or Plantae? due to other authors), 2. table 85 (fourth object in last column) a colonial ascidian (sea squirt) Cynthia of subphylum Tunicata to phylum Chordata of kingdom Animalia.

\section{References}

[1] Wünsche, A. (2014) Construction of Regular Heptagon by Rhombic Bicompasses and Ruler. Applied Mathematics, 5, 2370-2380. https://doi.org/10.4236/am.2014.515229

[2] Courant, R. and Robbins, H. (1996) What Is Mathematics. Oxford University Press, Oxford. (First Published 1941; I Used the Russian Translation from Prosvyetchenye, Moscow, 1967).

[3] van der Waerden, B.L. (1964) Algebra, 1. Teil. 6th Edition, Springer, Berlin. https://doi.org/10.1007/978-3-662-01380-9

[4] Postnikov, M.M. (1963) Teorija Galoa. Fizmatgiz, Moskva. (In Russian). (English Translation: Foundations of Galois Theory. Dover Publications, New York, 2004).

[5] Stewart, I. (2004) Galois Theory. 3rd Edition, Chapman \& Hall/CRC, Boca Raton.

[6] Edwards, H. (1984) Galois Theory. Springer, New York.

[7] Stillwell, J. (1994) Elements of Algebra. Springer, New York. https://doi.org/10.1007/978-1-4757-3976-3

[8] Stillwell, J. (2003) Elements of Number Theory. Springer, New York. https://doi.org/10.1007/978-0-387-21735-2

[9] Conway, J.H. and Guy, R.K. (1996) The Book of Numbers. Springer, New York; German Translation: Zahlenzauber, Birkhäuser, Basel, 1996.

[10] Stewart, I. and Tall, D. (2002) Algebraic Number Theory and Fermat's Last Theorem. CRC Press, Boca Raton.

[11] Shkolnik, A.G. (1961) The Problem of Circle Division. Uchpedgiz, Moscow. (In Russian).

[12] Bold, B. (1969) Famous Problems of Geometry and How to Solve Them. Dover, New York.

[13] Gleason, A.M. (1988) Angle Trisection, the Heptagon and the Triskaidecagon. The American Mathematical Monthly, 95, 185-195. https://doi.org/10.2307/2323624

[14] Gardner, M. (1966) The Persistence (and Futility) of Efforts to Trisect the Angle. Mathematical Games, Scientific American, June 1966. In: Matematicheskiye Novelly (Mathematical Stories) (in Russian) with a Collection of Articles from M. Gardner in Scientific American, Chap. 13, Mir, Moskva 1974.

[15] Gardner, M. (1969) Geometric Constructions with a Compass and Straightedge. Mathematical Games, Scientific American, September 1969. In: Matematicheskiye Novelly (Mathematical Stories) (in Russian) with a Collection of Articles from M. Gardner in Scientific American, Chap. 35, Mir, Moskva 1974.

[16] Szegö, G. (1950) Orthogonal Polynomials (Rev. Ed.). American Mathematical Society, New York.

[17] Rivlin, Th.J. (1990) Chebyshev Polynomials. 2nd Edition, John Wiley and Sons, New York. 
[18] Koornwinder, T.H., Wong, R., Koekoek, R. and Swarttouw, R.F. (2010) Orthogonal Polynomials. In: Olver, F.W.J., Lozier, D.W., Boisvert, R.F. and Clark, Ch.W., Eds., NIST Handbook of Mathematical Functions, Cambridge University Press, New York. Chap. 18, 435-484.

[19] Wikipedia Article Pierpont Prime, 2013 (with Historical Remarks to the Article).

[20] Weisstein, E.W. (2017) Pierpont Prime. From MathWorld-A Wolfram Web Resource. http://mathworld.wolfram.com/PierpontPrime.html

[21] Conway, J.H. and Smith, D.A. (2003) On Quaternions and Octonions. CRC Press, Boca Raton.

[22] Guy, R.K. (2004) Unsolved Problems in Number Theory. 3rd Edition, Springer, New York. https://doi.org/10.1007/978-0-387-26677-0 


\section{Appendix A. Two Linear Combinations of the Chebyshev Polynomials of First and Second Kind}

In Section 2 we came accross a class of polynomials for which sometimes the new notation $\mathrm{V}_{n}(x)$ is introduced. In this connection there are defined the following two relatives of the Chebyshev polynomials (see [16], p. 60 without notations $\mathrm{V}_{n}(x)$ and $\mathrm{W}_{n}(x)$ and, e.g., [18], p. 442)

$$
\begin{gathered}
\mathrm{V}_{n}(\cos (\theta))=\frac{\sin \left(\left(n+\frac{1}{2}\right) \theta\right)}{\sin \left(\frac{1}{2} \theta\right)}, \\
\mathrm{W}_{n}(\cos (\theta))=\frac{\cos \left(\left(n+\frac{1}{2}\right) \theta\right)}{\cos \left(\frac{1}{2} \theta\right)},
\end{gathered}
$$

called also Chebyshev polynomials of third and fourth kind. By multiplication of numerator and denominator of the first Equation in (A.1) with $\cos \left(\frac{1}{2} \theta\right)$ and of the second equation with $\sin \left(\frac{1}{2} \theta\right)$ and applying then the addition theorems for trigonometric functions one obtains

$$
\begin{gathered}
\mathrm{V}_{n}(\cos (\theta))=\frac{\sin ((n+1) \theta)+\sin (n \theta)}{\sin (\theta)}=\mathrm{U}_{n}(\cos (\theta))+\mathrm{U}_{n-1}(\cos (\theta)), \\
\mathrm{W}_{n}(\cos (\theta))=\frac{\sin ((n+1) \theta)-\sin (n \theta)}{\sin (\theta)}=\mathrm{U}_{n}(\cos (\theta))-\mathrm{U}_{n-1}(\cos (\theta)) .
\end{gathered}
$$

This can be also written in different representations (we use $\mathrm{T}_{-k}(z)=\mathrm{T}_{k}(z)$ and may use $U_{n-1}(z)=-U_{-n-1}(z)$ with special case $\left.U_{-1}(z)=0\right)$

$$
\begin{aligned}
\mathrm{V}_{n}(z) & =\frac{2^{2 n} n !^{2}}{(2 n) !} \mathrm{P}_{n}^{\left(\frac{1}{2},-\frac{1}{2}\right)}(z)=\frac{2^{2 n} n !^{2}}{(2 n) !}\left(\mathrm{P}_{n}^{\left(-\frac{1}{2},-\frac{1}{2}\right)}(z)+\frac{1+z}{2} \mathrm{P}_{n-1}^{\left(\frac{1}{2}, \frac{1}{2}\right)}(z)\right) \\
& =\mathrm{T}_{n}(z)+(1+z) \mathrm{U}_{n-1}(z)=\sum_{k=0}^{2 n} \mathrm{~T}_{n-k}(z)=\mathrm{U}_{n}(z)+\mathrm{U}_{n-1}(z), \\
\mathrm{W}_{n}(z)= & \frac{2^{2 n} n !^{2}}{(2 n) !} \mathrm{P}_{n}^{\left(-\frac{1}{2}, \frac{1}{2}\right)}(z)=\frac{2^{2 n} n !^{2}}{(2 n) !}\left(\mathrm{P}_{n}^{\left(-\frac{1}{2},-\frac{1}{2}\right)}(z)-\frac{1-z}{2} \mathrm{P}_{n-1}^{\left(\frac{1}{2}, \frac{1}{2}\right)}(z)\right) \\
= & \mathrm{T}_{n}(z)-(1-z) \mathrm{U}_{n-1}(z)=\sum_{k=0}^{2 n}(-1)^{k} \mathrm{~T}_{n-k}(z)=\mathrm{U}_{n}(z)-\mathrm{U}_{n-1}(z),
\end{aligned}
$$

where $\mathrm{P}_{n}^{(\alpha, \beta)}(z)$ denotes the Jacobi polynomials.

The orthogonality relations and weight functions for the polynomials $\mathrm{V}_{n}(x)$ and $\mathrm{W}_{n}(x)$ in the real interval $-1 \leq x \leq+1$ are different from that for the Chebyshev polynomials $\mathrm{T}_{n}(x)$ and $\mathrm{U}_{n}(x)$ and follow from the general orthogonality relations for the Jacobi polynomials. The weight functions are 
$\sqrt{\frac{1-x}{1+x}}$ and $\sqrt{\frac{1+x}{1-x}}$ for $\mathrm{V}_{n}(x)$ and $\mathrm{W}_{n}(x)$, respectively, instead of $\frac{1}{\sqrt{1-x^{2}}}$ and $\sqrt{1-x^{2}}$ for $\mathrm{T}_{n}(x)$ and $\mathrm{U}_{n}(x)$, respectively. This circumstance may justify the separate introduction of $\mathrm{V}_{n}(x)$ and $\mathrm{W}_{n}(x)$ but most relations for these polynomials can be directly obtained from that for the Chebyshev polynomials of first and second kind.

Submit or recommend next manuscript to SCIRP and we will provide best service for you:

Accepting pre-submission inquiries through Email, Facebook, LinkedIn, Twitter, etc. A wide selection of journals (inclusive of 9 subjects, more than 200 journals)

Providing 24-hour high-quality service

User-friendly online submission system

Fair and swift peer-review system

Efficient typesetting and proofreading procedure

Display of the result of downloads and visits, as well as the number of cited articles

Maximum dissemination of your research work

Submit your manuscript at: http://papersubmission.scirp.org/

Or contact apm@scirp.org 